\title{
Matter waves in two-dimensional arbitrary atomic crystals
}

\author{
Nicola Bartolo ${ }^{1,2,3, *}$ and Mauro Antezza ${ }^{1,2,4, \dagger}$ \\ ${ }^{1}$ Université Montpellier 2, Laboratoire Charles Coulomb UMR 5221, F-34095 Montpellier, France \\ ${ }^{2}$ CNRS, Laboratoire Charles Coulomb UMR 5221, F-34095 Montpellier, France \\ ${ }^{3}$ INO-CNR BEC Center and Dipartimento di Fisica, Università di Trento, I-38123 Povo, Italy \\ ${ }^{4}$ Institut Universitaire de France, 103 bd Saint-Michel, F-75005 Paris, France \\ (Received 28 February 2014; revised manuscript received 19 July 2014; published 17 September 2014)
}

\begin{abstract}
We present a general scheme to realize a cold-atom quantum simulator of bidimensional atomic crystals. Our model is based on the use of two independently trapped atomic species: the first one, subject to a strong in-plane confinement, constitutes a two-dimensional matter wave which interacts only with atoms of the second species, deeply trapped around the nodes of a two-dimensional optical lattice. By introducing a general analytic approach we show that the system Green function can be exactly determined, allowing for the investigation of the matter-wave transport properties. We propose some illustrative applications to both Bravais (square, triangular) and non-Bravais (graphene, kagomé) lattices, studying both ideal periodic systems and experimental-size and disordered ones. Some remarkable spectral properties of these atomic artificial lattices are pointed out, such as the emergence of single and multiple gaps, flat bands, and Dirac cones. All these features can be manipulated via the interspecies interaction, which proves to be widely tunable due to the interplay between scattering length and confinements.
\end{abstract}

DOI: 10.1103/PhysRevA.90.033617

PACS number(s): 03.75.-b, 37.10.Jk, 67.10.Jn, 67.85.-d

\section{INTRODUCTION}

In spite of the development of supercomputers and cuttingedge numerical methods, the simulation of experimental-size many-body systems is still a hard task. Following Feynman's conjecture of a quantum simulator, it proves useful to analyze manipulable systems able to reproduce, in a controllable way, the physical scenario that one wants to investigate. The theoretical and experimental progress of the last decades, boosted by nanotechnological needs, has made it possible to combine the properties of cold gases and optical lattices to build up artificial crystals able to mimic condensed-matter systems [1]. A crucial factor in the success of these models is their experimental versatility. Interactions between cold atoms can indeed be tuned via Feshbach [2], dipolar-induced [3], or confinement-induced [4] resonances. On the other hand, optical lattices offer full control of the potential landscape felt by cold atoms, allowing for the exploration of quantum phase transitions [5]. Furthermore, these kinds of simulators enable the exploration of parameter ranges beyond those of the real material they imitate, unveiling new physical scenarios.

In solid-state physics, among the plethora of crystals that can be investigated, two-dimensional ones (2D) are of special interest due to the intriguing properties that $2 \mathrm{D}$ materials have been shown to possess. Up to the early 2000s the study of these systems was only of academic interest, 2D solids being considered unstable structures never observed experimentally. Things changed in 2004, when graphene was finally isolated [6]. This discovery paved the way to the study of this astonishing carbon allotrope composed of a monolayer of ions forming a honeycomb lattice in which charge carriers manifest peculiar transport properties [7,8]. In particular, conduction and valence bands touch in isolated points of $k$

\footnotetext{
*nicola.bartolo@univ-montp2.fr

†mauro.antezza@univ-montp2.fr
}

space: the Dirac points. Around them the energy-momentum dispersion relation is conical and a Dirac-like equation for massless fermions replaces Schrödinger's equation to describe the quantum motion of the carriers. Graphene thus qualifies as a quantum electrodynamics simulator on a benchtop scale. Furthermore, relativistic effects, in general, inversely proportional to the speed of light, would be enhanced in graphene: the role of $c$ is played here by the group velocity $v_{g}$ of the particles around the cone and $c / v_{g} \sim 300$ [7].

The growing attention to graphene and other monolayer materials translates into an increasing interest in their quantum simulators, so that many artificial prototypes of 2D lattices have been proposed and realized in past years (for a recent review see [9]). In the present work we introduce a general, highly controllable model for the realization of artificial bidimensional lattices, based on the use of two cold-atomic species. In our system a 2D matter wave (MW), made up of $A$ atoms, interacts with point-like scatterers of the second atomic species, denoted $B$, independently trapped around the nodes of a 2D optical lattice. A schematic of our model is presented in Fig. 1. Such a scheme is already experimentally realizable using species-selective optical lattices: trapping potentials engineered to act on an atomic species ( $B$ in the present case) being, at the same moment, invisible to a second one (for us, A) [10,11]. This has been done, for instance, in Ref. [10] for a mixture of ${ }^{87} \mathrm{Rb}$ and ${ }^{41} \mathrm{~K}$ atoms: when the optical-lattice frequency is tuned exactly in between two ${ }^{87} \mathrm{Rb}$ resonances, the attractive and repulsive contributions to the optical force cancel each other and only ${ }^{41} \mathrm{~K}$ feels the added potential. In this study we limit our investigations to one-body physics in the MW; i.e., we assume $A$ atoms to be noninteracting with themselves, a situation attainable by using polarized fermions or bosons at zero scattering length. One can instead employ the $B-B$ interaction to reach a Mott insulating phase with exactly one atom per lattice site [5] and subsequently freeze the atoms in this configuration by increasing the lattice depth. Other techniques are also available 


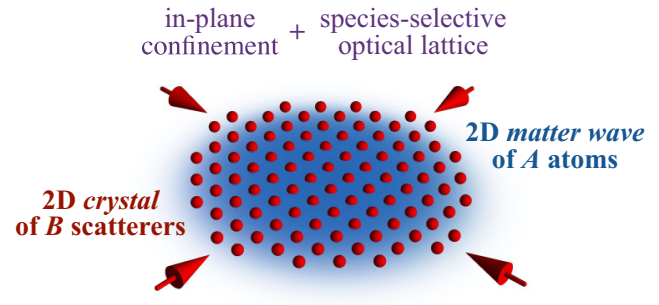

FIG. 1. (Color online) Schematic of our model for the realization of $2 \mathrm{D}$ atomic crystals. Two atomic species, namely, $A$ (depicted in blue) and $B$ (depicted in red), are strongly confined on a plane. Making use of a species-selective optical lattice [10,11], $B$ atoms are arranged in a 2D lattice of point-like scatterers of arbitrary geometry (a square one in this example), while $A$ atoms form a matter wave which propagates through the artificial crystal.

to probe [12] and manipulate [13], at the single-site and single-atom level, the scatterer arrangement. This model has recently been proposed to study the effects of disorder in 1D [14], 2D, and 3D [15] systems, and it has the advantage of showing a one-to-one correspondence with the bidimensional lattices that it can mimic : the $A$ atoms of the MW play the role of the electronic cloud, while the deeply trapped $B$ atoms represent the crystalline structure. Henceforth we refer to our system as an atomic artificial crystal (AAC), since the periodic potential felt by the MW is generated by other atoms and not by an optical potential.

The paper is organized as follows. We start by introducing the theoretical model in Sec. II, briefly discussing the problem of scattering in reduced and mixed dimensions (Sec. II A) and describing the general approach to the study of an AAC in Sec. IIB. In Sec. III we specify the model to the case of ideal Bravais arrangements of the scatterers, studying as illustrative examples the square (Sec. III A) and triangular (Sec. III B) lattices. For both of them the spectral properties are analyzed as a function of the $A-B$ interaction strength. Finite-size and disorder effects are also investigated. Sec. IV is devoted to the generalization of previous results to non-Bravais lattices, focusing on the exemplary atomic artificial graphene (AAG) (Sec. IV A) [16] and kagomé lattice (Sec. IV B). Some properties of these systems, namely, the emergence of Dirac cones and nondispersive flat bands, are pointed out and characterized. We, finally, present our conclusions in Sec. V.

\section{THEORETICAL MODEL}

\section{A. 0D-2D scattering process}

A remarkable feature of cold-atomic systems is the possibility of using experimentally controllable parameters as knobs to tune the interatomic scattering lengths, for example, by means of Feshbach or dipolar-induced resonances [2,3]. When the interacting particles are subjected to a trapping potential, this can, in turn, play a role leading to confinementinduced resonances [4]. If the trapping is sufficiently strong, the dimensionality of the system can be reduced: it has been proven, for instance, that a $3 \mathrm{D}$ system subject to a strong axial confinement (quasi-2D) can be mapped into a strictly $2 \mathrm{D}$ one by introducing an effective $2 \mathrm{D}$ scattering
Actual scattering process in 3D

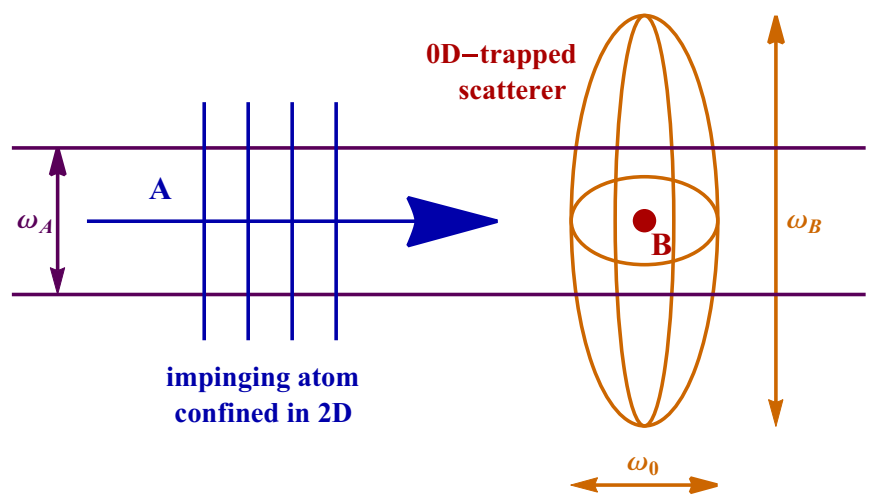

Equivalent scattering process in 2D

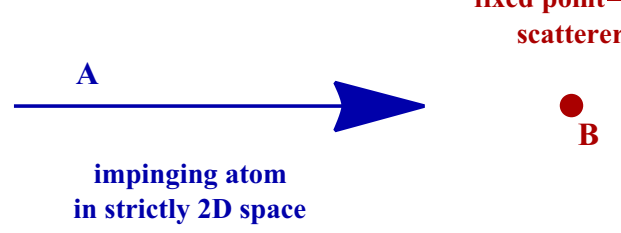

FIG. 2. (Color online) Schematic of the two-body scattering process between a 2D-trapped $A$ atom and a 0D-trapped $B$ atom. The scattering process in the actual system (top) is ruled by the $3 \mathrm{D} A-B$ scattering length $a_{3 \mathrm{D}}$ of the contact interaction, as well as by the trapping frequencies and the mass ratio of the two atomic species. The system is equivalent to a strictly bidimensional one (bottom) in which the $B$ atom constitutes a fixed point-like scatterer and the scattering process is ruled only by an effective $2 \mathrm{D}$ scattering length $a_{2 \mathrm{D}}^{\text {eff }}[20]$.

length, the latter depending on the free 3D interaction and on the trapping parameters [17]. Another related subject is the study of scattering processes involving differently trapped atoms; in this case we talk about scattering in mixed dimensions. Recently processes involving a free particle and a trapped one have been addressed $(n \mathrm{D}-3 \mathrm{D}$ scattering $[18,19])$ and some theoretical predictions have been tested experimentally [10].

In the case of the AAC the building block of any analysis is the two-body low-energy scattering process between an $A$ atom of mass $m_{A}=m$, harmonically trapped on a plane, and a $B$ one of mass $m_{B}$, trapped around a node of an optical lattice, the process represented schematically in Fig. 2. It can be shown that, by providing a proper effective $2 \mathrm{D}$ scattering length $a_{2 \mathrm{D}}^{\text {eff }}$, the system is mapped into a strictly $2 \mathrm{D}$ space in which the $B$ atom is now a fixed point-like scatterer. The effective parameter $a_{2 \mathrm{D}}^{\text {eff }}$, which also takes into account the quantum motion of the $B$ atom in the real system, depends on the atomic mass ratio, the trapping frequencies, and the $A-B$ scattering length $a_{3 \mathrm{D}}$ in free space (cf. Fig. 2). A detailed study of this 0D-2D scattering process will be extensively presented elsewhere [20]. For the purposes of the present work we assume $a_{2 \mathrm{D}}^{\text {eff }}$ to be tunable in its full range of existence $[0, \infty$ [, protecting the validity of the point-like approximation for $B$. In this regime the interaction can be taken into account by considering the $A$ atom as free 
and imposing the Bethe-Peierls contact conditions,

$$
\psi(\mathbf{r}) \stackrel{\mathbf{r} \rightarrow \mathbf{r}_{B}}{\longrightarrow} \frac{m}{\pi \hbar^{2}} D_{B} \ln \left(\frac{\left|\mathbf{r}-\mathbf{r}_{B}\right|}{a_{2 \mathrm{D}}^{\text {eff }}}\right)+O\left(\left|\mathbf{r}-\mathbf{r}_{B}\right|\right),
$$

on its wave function at the position $\mathbf{r}_{B}$ of the scatterer, where $D_{B}$ is an arbitrary complex coefficient. We, finally, recall that, in the case of 2D scattering, the limit $a_{2 \mathrm{D}}^{\text {eff }} \rightarrow \infty$ corresponds to a weakly attractive interaction, while for $a_{2 \mathrm{D}}^{\text {eff }} \rightarrow 0$ the effective potential allows a single, infinitely deep bound state and results weakly repulsive for positive-energy scattering states [21].

\section{B. General approach}

Let us start by considering now a general case in which $N$ point-like $B$ scatterers are fixed at positions $\left\{\mathbf{r}_{i}\right\}$. The first steps of our calculation follow [15]: we consider the Hamiltonian of the MW as that of a free $A$ atom, i.e., $\mathcal{H}=-\frac{\hbar^{2}}{2 m} \nabla_{2 \mathrm{D}}^{2}$, with $\nabla_{2 \mathrm{D}}^{2}$ the 2D Laplace operator, and add the $A-B$ interaction by imposing the boundary condition (1) at the position of each $B$ scatterer. This introduces a set of $N$ independent complex coefficients $D_{i}$. The same conditions apply to the MW Green's function, $G\left(\mathbf{r}, \mathbf{r}_{0}\right)$, solution of the Schrödinger equation for a point-like source term of MWs in $\mathbf{r}_{0}:\left(E+i 0^{+}-\mathcal{H}\right) G\left(\mathbf{r}, \mathbf{r}_{0}\right)=\delta\left(\mathbf{r}-\mathbf{r}_{0}\right)$. The latter wave equation can be rewritten to take the boundary conditions directly into account. By using the identity $\nabla_{2 \mathrm{D}}^{2} \ln (r)=2 \pi \delta(\mathbf{r})$, the effect of contact conditions resumes in the inclusion of secondary point-like sources of amplitude $D_{i}$ at the position of each scatterer [15], leading to

$$
\left(E+i 0^{+}-\mathcal{H}\right) G\left(\mathbf{r}, \mathbf{r}_{0}\right)=\delta\left(\mathbf{r}-\mathbf{r}_{0}\right)+\sum_{i=1}^{N} D_{i} \delta\left(\mathbf{r}-\mathbf{r}_{i}\right) .
$$

Since the poles of $G$ (and of its analytical continuation to complex energies in the lower half-plane) correspond to eigenstates of the system, its knowledge is of fundamental importance to determine the properties of the AAC.

To integrate Eq. (2) we use its solution in the absence of scatterers, which is the case for a free 2D MW. In this case, $G\left(\mathbf{r}, \mathbf{r}_{0}\right)=g\left(\mathbf{r}-\mathbf{r}_{0}\right)$, with

$$
g_{0}(\mathbf{r})=-i \frac{m}{2 \hbar^{2}} H_{0}^{(1)}(k r),
$$

where $H_{0}^{(1)}$ is the zero-index Hankel function of the first kind. The wave-vector modulus $k$ is linked to the MW energy by $E=\hbar^{2} k^{2} / 2 m$, with $k>0$ for $E>0$ and $k=i \kappa$ with $\kappa>0$ for $E<0$ (i.e., for bound states). The formal solution of the wave equation is hence

$$
G\left(\mathbf{r}, \mathbf{r}_{0}\right)=g_{0}\left(\mathbf{r}-\mathbf{r}_{0}\right)+\sum_{i=1}^{N} D_{i} g_{0}\left(\mathbf{r}-\mathbf{r}_{i}\right),
$$

where the determination of the $N$ coefficients $D_{i}$ depends on the system geometry, encoded in the set $\left\{\mathbf{r}_{i}\right\}$. This problem can be traced back to the solution of a complex linear system in the $N$ unknowns $D_{i}$. Each equation of this system comes from the limit $\mathbf{r} \rightarrow \mathbf{r}_{j}$ of Eq. (4), imposing the Bethe-Peierls condition on the left-hand side and applying

$$
H_{0}^{(1)}(k r) \underset{r \rightarrow 0}{\longrightarrow} 1+\frac{2 i}{\pi} \ln \left(\frac{e^{\gamma}}{2} k r\right)+o(1)
$$

on the right-hand side, where $\gamma \simeq 0.577216$ is the EulerMascheroni constant. After some straightforward algebraic manipulation the system can be cast as

$$
\sum_{i=1}^{N} \mathbb{M}_{j i} D_{i}=-\frac{\pi \hbar^{2}}{m} g_{0}\left(\mathbf{r}_{j}-\mathbf{r}_{0}\right) \quad j=1,2, \ldots, N,
$$

with the introduction of the matrix $\mathbb{M}$ of elements

$$
\mathbb{M}_{j i}= \begin{cases}\frac{\pi \hbar^{2}}{m} g_{0}\left(\mathbf{r}_{j}-\mathbf{r}_{i}\right), & \mathbf{r}_{j} \neq \mathbf{r}_{i} \\ \ln \left(\frac{e^{\gamma}}{2} k a_{2 \mathrm{D}}^{\text {eff }}\right)-i \frac{\pi}{2}, & \mathbf{r}_{j}=\mathbf{r}_{i} .\end{cases}
$$

The formal solution of $G$ [Eq. (4)] has a pole if $\mathbb{M}$ is not invertible, i.e., if $\operatorname{det}(\mathbb{M})=0$. The latter is thus our general condition for the existence of an eigenstate of the MW in the gas of scatterers.

The condition $\operatorname{det}(\mathbb{M})=0$ can be rewritten in a more practical form by noting that the interaction-dependent terms appear only in the diagonal elements of $\mathbb{M}$. In particular, one can write

$$
\mathbb{M}_{j j}=\ln \left(\frac{e^{\gamma}}{2} k a\right)-i \frac{\pi}{2}+\alpha,
$$

with the introduction of the 2D interaction coefficient $\alpha=$ $\ln \left(a_{2 \mathrm{D}}^{\text {eff }} / a\right)$ and for an arbitrary choice of the unitary length $a$. It follows that $\mathbb{M}=\mathbb{M}^{o}+\mathbb{I} \alpha$, for $\mathbb{M}^{o}=\mathbb{M}(\alpha=0)$ and $\mathbb{I}$ the $N \times N$ identity matrix. For $E<0$ the matrix $\mathbb{M}^{o}$ is real, and looking for solutions of $\operatorname{det}(\mathbb{M})=0$ is equivalent to solving

$$
m_{i}^{o}(E)=-\alpha, \quad i=1,2, \ldots, N,
$$

for each of the $N$ eigenvalues $m_{i}^{o}$ of $\mathbb{M}^{o}$. Solutions of Eq. (9) give real and negative energies of the MW bound states in the gas of scatterers. For $E>0$ the situation is slightly different. A continuum of states is allowed for the MW; nevertheless, for a large enough number of scatterers, precursors of the bulk Bloch states of the infinite periodic system can be identified in the form of complex poles of the analytical continuation of $G$ to the lower half-plane of complex energies. In our approach, this corresponds to the fact that $\mathbb{M}^{o}$ is now a complex matrix and the poles of the extended $G$ can be found by solving

$$
m_{i}^{o}(z)=-\alpha \quad i=1,2, \ldots, N,
$$

for complex energies of the form $z=E-i \hbar \Gamma / 2$, where $E$ and $\Gamma>0$ represent, respectively, the position and bandwidth (i.e., inverse lifetime) of the eigenstate. The latter, in an extended and ordered system, would be a quasi-Bloch state, i.e., a state showing the periodicity properties of a Bloch state within the gas of scatterers but with a finite lifetime inside of it.

\section{BRAVAIS LATTICES}

In this section we adapt the general formalism introduced in Sec. II B to the case in which $B$ atoms are arranged in a Bravais lattice: an infinite periodic structure where a unit cell, containing only one atom, is repeated to cover the entire 2D space (cf. Figs. 3 and 4). Such a lattice is invariant under any translation $\mathbf{R} \in L$ with $L=\left\{n_{1} \mathbf{a}_{1}+n_{2} \mathbf{a}_{2}: n_{1}, n_{2} \in \mathbb{Z}\right\}$, where the set $L$ is defined in terms of the two primitive vectors $\mathbf{a}_{1}$ and $\mathbf{a}_{2}$. Consequently, we can define the reciprocal lattice as a periodic structure invariant under translations $\mathbf{K} \in R L$ with $R L=\left\{n_{1} \mathbf{b}_{1}+n_{2} \mathbf{b}_{2}: n_{1}, n_{2} \in \mathbb{Z}\right\}$, where $\mathbf{b}_{1}$ and $\mathbf{b}_{2}$ are 

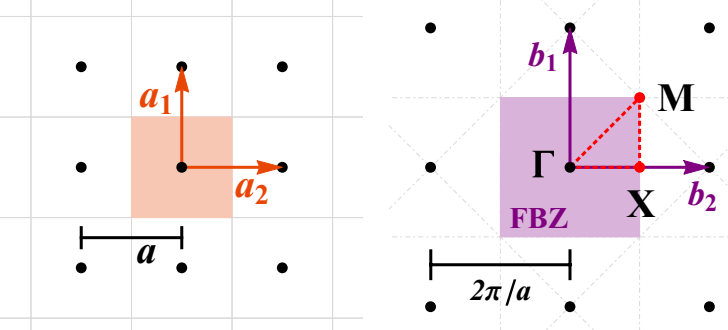

FIG. 3. (Color online) Left: Schematic in real space of a square lattice of primitive vectors $\mathbf{a}_{1}=a(1,0)$ and $\mathbf{a}_{2}=a(0,1)$. The shaded area represents the unit cell. Right: Reciprocal lattice in $k$ space corresponding to the square lattice. Consequently the reciprocal primitive vectors are $\mathbf{b}_{1}=\frac{2 \pi}{a}(1,0)$ and $\mathbf{b}_{2}=\frac{2 \pi}{a}(0,1)$. The first Brillouin zone (FBZ) is shaded. The high-symmetry points $\boldsymbol{\Gamma}=(0,0), \mathbf{X}=\frac{\pi}{a}(1,0)$, and $\mathbf{M}=\frac{\pi}{a}(1,1)$ are highlighted and the $\boldsymbol{\Gamma}-\mathbf{X}-\mathbf{M}-\boldsymbol{\Gamma}$ path [dashed (red) line] constitutes an irreducible symmetry path.

the reciprocal primitive vectors, defined by the relation $\mathbf{a}_{i}$. $\mathbf{b}_{j}=2 \pi \delta_{i j}(i, j=1,2)$ [22]. Some examples of Bravais and corresponding reciprocal lattices are presented in Figs. 3 and 4.

The condition for the existence of an eigenstate, i.e., $\operatorname{det}(\mathbb{M})=0$, implies that the homogeneous system

$$
\sum_{i=1}^{\infty} \mathbb{M}_{j i} D_{i}=0, \quad j=1,2, \ldots, \infty,
$$

associated with the inhomogeneous one of Eq. (6) admits a nontrivial solution. Note that now the number of scatterers $N$, and thus the number of equations and unknowns in the system, is infinite [23,24]. In such a periodic structure Bloch's theorem holds, implying that

$$
D_{i}=D_{j} e^{i \mathbf{q} \cdot\left(\mathbf{r}_{j}-\mathbf{r}_{i}\right)},
$$

where $\mathbf{q}$ is a vector of the first Brillouin zone (FBZ) in reciprocal space. Resorting to this property all the equations of the homogeneous system (11) become identical, so that the
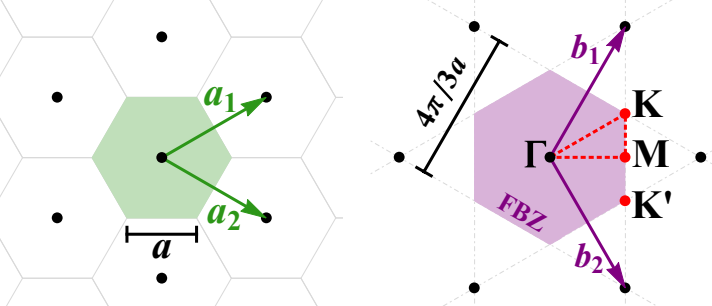

FIG. 4. (Color online) Left: Real-space representation of a triangular lattice of primitive vectors $\mathbf{a}_{1}=\frac{3 a}{2}(1,1 / \sqrt{3})$ and $\mathbf{a}_{2}=\frac{3 a}{2}(1$, $-1 / \sqrt{3})$. The shaded region represents the Wigner-Seitz unit cell. Right: Reciprocal lattice for the triangular one, $\mathbf{b}_{1}=\frac{2 \pi}{3 a}(1, \sqrt{3})$ and $\mathbf{b}_{2}=\frac{2 \pi}{3 a}(1,-\sqrt{3})$ being the reciprocal primitive vectors. The first Brillouin zone (FBZ) is shaded and the high-symmetry points $\boldsymbol{\Gamma}=(0,0), \mathbf{M}=\frac{2 \pi}{3 a}(1,0), \mathbf{K}=\frac{2 \pi}{3 a}(1,1 / \sqrt{3})$, and $\mathbf{K}^{\prime}=\frac{2 \pi}{3 a}(1,-1 / \sqrt{3})$ are highlighted. The $\boldsymbol{\Gamma}-\mathbf{M}-\mathbf{K}-\boldsymbol{\Gamma}$ path [dashed (red) line] is a highsymmetry one. unique condition to verify is

$$
\ln \left(\frac{e^{\gamma}}{2} k a_{2 \mathrm{D}}^{\mathrm{eff}}\right)-i \frac{\pi}{2}+\sum_{\mathbf{R} \in L^{*}} \frac{\pi \hbar^{2}}{m} g_{0}(\mathbf{R}) e^{i \mathbf{q} \cdot \mathbf{R}}=0,
$$

where $L^{*}=L \backslash\{0\}$. Due to the slow convergence of the sum in Eq. (13), a rewriting of the equation in terms of reciprocal-lattice vectors is appropriate. The delicate details of this transformation are reported in the Appendix 1, the result being

$$
\begin{aligned}
C_{\infty} & +\ln \left(\frac{e^{\gamma}}{2}\right)+\frac{2 \pi}{\mathcal{A}} \frac{1}{k^{2}-q^{2}} \\
& +\frac{2 \pi}{\mathcal{A}} \sum_{\mathbf{K} \in R L^{*}}\left(\frac{1}{k^{2}-|\mathbf{K}-\mathbf{q}|^{2}}+\frac{1}{K^{2}}\right)+\alpha=0 .
\end{aligned}
$$

Here $C_{\infty}$ is a coefficient depending only on the geometry of the Bravais lattice; its origin and definition follow from the real-to-reciprocal lattice transformation, presented in the Appendix 1. We have also reintroduced the 2D interaction coefficient $\alpha=\ln \left(a_{2 \mathrm{D}}^{\text {eff }} / a\right)$ ( $a$ being an arbitrary unit of length) and $\mathcal{A}$ is the area of the real-space unit cell of the Bravais lattice.

It is worth noting that Eq. (14) can be cast as

$$
f(\mathbf{q}, E)=-\alpha,
$$

with the introduction of the interaction-independent function

$$
\begin{aligned}
f(\mathbf{q}, E)= & C_{\infty}+\ln \left(\frac{e^{\gamma}}{2}\right)+\frac{2 \pi}{\mathcal{A}} \frac{1}{k^{2}-q^{2}} \\
& +\frac{2 \pi}{\mathcal{A}} \sum_{\mathbf{K} \in R L^{*}}\left(\frac{1}{k^{2}-|\mathbf{K}-\mathbf{q}|^{2}}+\frac{1}{K^{2}}\right) .
\end{aligned}
$$

It follows by its definition that $f(\mathbf{q}, E)$ diverges for

$$
k=|\mathbf{K}-\mathbf{q}| \quad \forall \mathbf{K} \in R L,
$$

where wave vector and energy of the MW are related by $E=\hbar^{2} k^{2} / 2 m$. Furthermore, $f(\mathbf{q}, E)$ is monotonically decreasing in $E$ between two divergences, ensuring that for a given $\mathbf{q}$ only one solution of Eq. (15) exists between them. Another remarkable consequence is that if Eq. (17) holds, then condition (15) is satisfied only for $|\alpha| \rightarrow \infty$, which is in the limit of noninteraction between the MW and scatterers (cf. Fig. 5 for a practical example). This implies that for $|\alpha| \rightarrow \infty$ one would recover the dispersion relation of a free MW, whose energy is given by

$$
E_{\text {free }}=\frac{\hbar^{2}}{2 m}|\mathbf{K}-\mathbf{q}|^{2} \quad \forall \mathbf{K} \in R L .
$$

\section{A. Square lattice}

As the first example of an AAC we consider $B$ scaterers arranged in a square lattice of spacing $a$, for which the primitive vectors are simply $\mathbf{a}_{1}=a(1,0)$ and $\mathbf{a}_{2}=a(0,1)$. The reciprocal lattice and the FBZ are consequently defined, as illustrated in Fig. 3.

\section{Infinite system}

To study the band structure of the artificial atomic square lattice (AASL) one needs to solve Eq. (14), where in this 


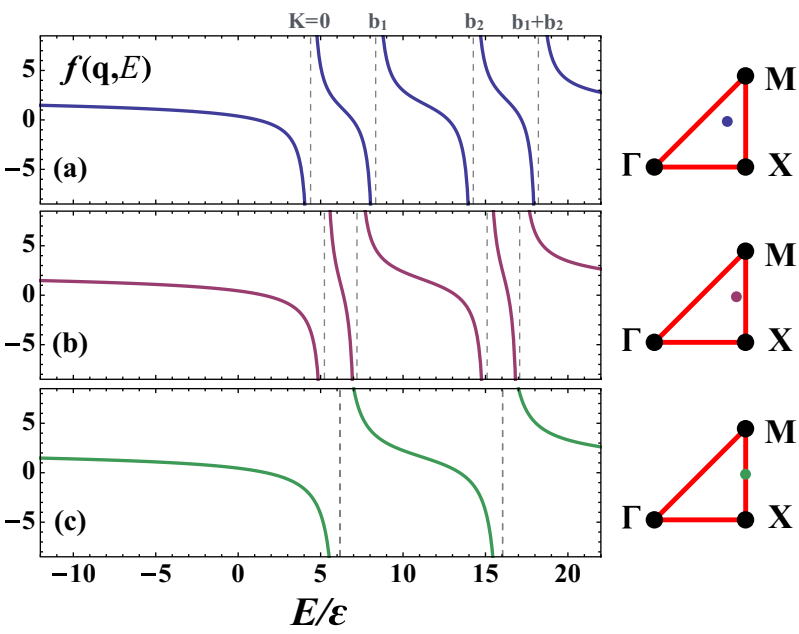

FIG. 5. (Color online) For a square lattice (cf. Fig. 3), behavior of $f(\mathbf{q}, E)$ as a function of $E$ for different values of $\mathbf{q}$ [namely, $\frac{\pi}{a}(0.8,0.5), \frac{\pi}{a}(0.9,0.5)$, and $\frac{\pi}{a}(1,0.5)$, from top to bottom]. Dashed vertical (gray) lines represent the lowest values of $E_{\text {free }}$ for the selected q. In the sketches at the right of each panel a point indicates the corresponding $\mathbf{q}$ inside the $\boldsymbol{\Gamma}-\mathbf{X}-\mathbf{M}-\boldsymbol{\Gamma}$ symmetry path. Here $\varepsilon=\hbar^{2} / m a^{2}$.

case $C_{\infty} \simeq 1.42646$ [cf. Eq. (A12)]. It is convenient from a computational point of view to evaluate once and for all the function $f(\mathbf{q}, E)$ defined in Eq. (16) and then look for solutions of Eq. (15) for a given $\alpha$. In Fig. 5 we plot $f(\mathbf{q}, E)$ for different values of $\mathbf{q}$ inside the FBZ. As expected, $f$ diverges each time that $E=E_{\text {free }}$ [i.e., when condition (17) is satisfied], but when $\mathbf{q}$ gets closer to the boundary of the FBZ some values of $E_{\text {free }}$ corresponding to different $\mathbf{K}$ eventually tend to each other. A solution of Eq. (15) thus remains "trapped" in the small corridor formed by the two divergences and it tends to $E=E_{\text {free }}$ in the degeneracy limit, independently of $\alpha$.

We present in Fig. 6 the spectrum and density of states (DOS) of the AASL for $\alpha=-0.75$. The spectrum is evaluated along the $\boldsymbol{\Gamma}-\mathbf{X}-\mathbf{M}-\boldsymbol{\Gamma}$ symmetry path (cf. inset). The free-MW

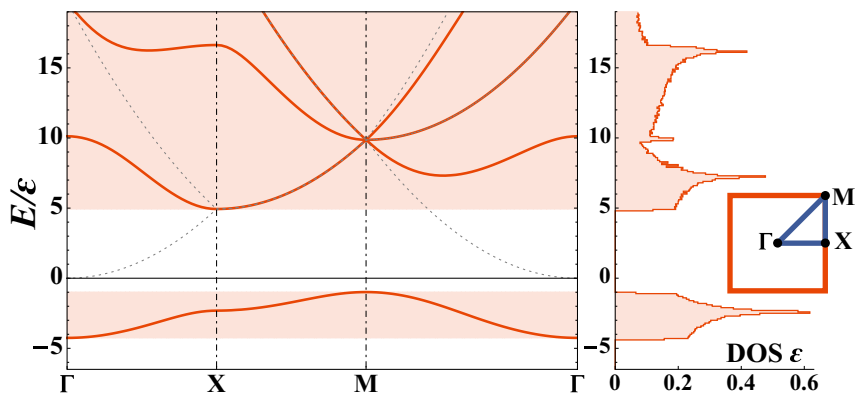

FIG. 6. (Color online) For the artificial atomic square lattice, the band structure (left) and density of states (right) evaluated at $\alpha=$ -0.75 . Left: The four lowest energy bands are evaluated along the irreducible symmetry path $\boldsymbol{\Gamma}-\mathbf{X}-\mathbf{M}-\boldsymbol{\Gamma}$ within the first Brilouin zone (see inset). Dashed (gray) lines correspond to the energy spectrum of a free matter wave. Right: Density of states (DOS) obtained by evaluating the energies of the same bands in $N_{s} \simeq 6100$ points sampled inside the path. For each bin of the histogram (of width $\delta E=0.1 \varepsilon, \varepsilon=$ $\hbar^{2} / m a^{2}$ ) the band-normalized DOS is evaluated according to Eq. (19).

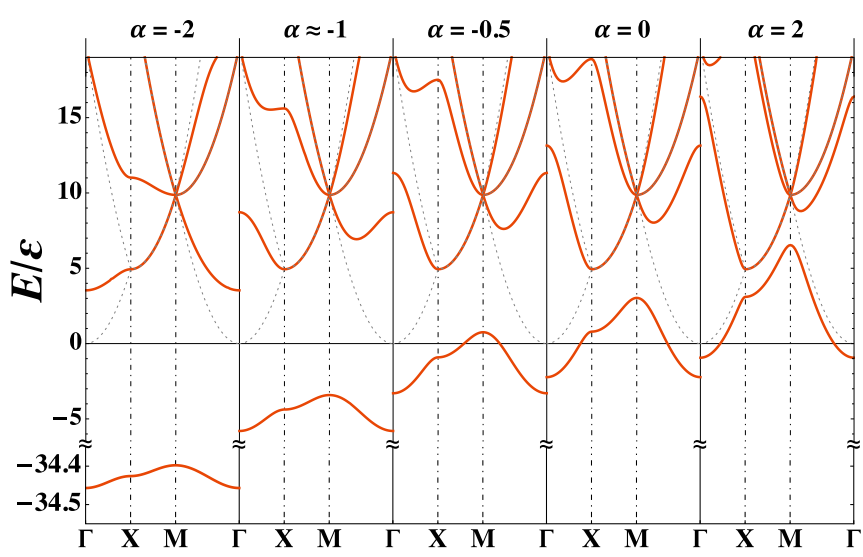

FIG. 7. (Color online) Square lattice: band structure for different values of the interaction parameter $\alpha$. Spectra are evaluated along the same path as shown in Fig. 6. Dashed (gray) lines correspond to the energy spectrum of a free matter wave. Energies are normalized on $\varepsilon=\hbar^{2} / m a^{2}$.

energy $E_{\text {free }}$ is also shown, and as expected, only one solution of Eq. (15) exists between two (eventually coinciding) free bands. An omnidirectional gap is found in the band structure and, correspondingly, in the DOS. The numerical evaluation of the DOS can be obtained by sampling each energy band in $N_{s}$ points within the FBZ. Subsequently, the formula

$$
\operatorname{DOS}(E)=\frac{N_{E}}{N_{s} \delta E}
$$

gives the DOS in the energy range $(E, E+\delta E), N_{E}$ being the number of sampled energies falling in the energy interval. The DOS defined in Eq. (19) is normalized to the number of bands taken into account. Finally, we remark that the lowest, isolated band lies entirely at negative energies for this value of $\alpha$, meaning that the MW Bloch states are actually bound.

A fundamental aspect of our model relies on the possibility of tuning its physical properties by acting on $\alpha$. This remarkable feature is shown in Fig. 7, where the spectra of the AASL are compared for different values of $\alpha$. The most evident modification concerns the lowest band, which is isolated for $\alpha \lesssim 0.7453$, so that a gap opens in the spectrum. Furthermore, the band becomes increasingly deep and flat, leading to low group velocities for the corresponding eigenstates. For $|\alpha| \gg 1$ the MW and scatterers are weakly interacting, and as expected, the band structure tends to the free-MW spectrum [dashed (gray) curves in Fig. 7].

\section{Finite-size effects}

Up to now we have considered ideal periodic systems, but for both theoretical and practical interests it is crucial to investigate the robustness of the above-reported results for realistic finite-size systems. In this case one cannot resort to Bloch's theorem and the use of the general approach presented in Sec. II B is necessary. In a typical experimental setup, atomic clouds can be manipulated in optical lattices extending over $\sim 60$ sites per dimension. This means that $2 \mathrm{D}$ artificial atomic lattices with $\sim 10^{3}$ trapped $B$ scatterers are experimentally realizable. In Fig. 8 we thus present the DOS on the $[\alpha, E]$ plane for a finite system of $\sim 2500$ scatterers arranged in a 


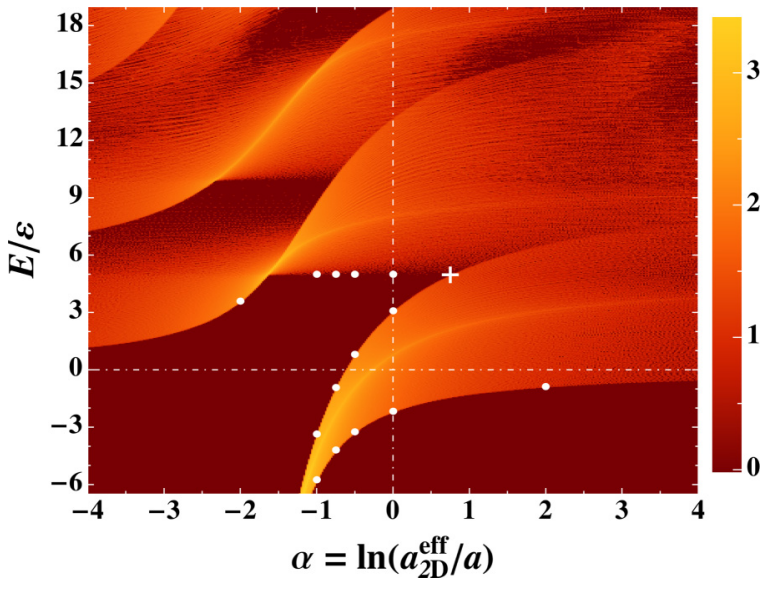

FIG. 8. (Color online) Finite-sized square lattice: DOS per scatterer in the plane $[\alpha, E / \varepsilon]$ for a system of $N=2551$ scatterers arranged in a square lattice inside a disk of radius $R=26 \mathrm{a}$. Energies are discretized with a step of $0.01 \varepsilon\left(\varepsilon=\hbar^{2} / m a^{2}\right)$. For a given $E<0$ all $N$ solutions of Eqs. (9) are selected. For $E>0$ the sampled values are used as starting points to find $N$ complex poles of $G$ according to Eq. (20). Only quasi-Bloch bulk states are selected, as explained in the text. The color map is applied to $\log _{10}\left(\frac{N_{p}}{N} \frac{\varepsilon}{\delta \alpha \delta E}\right)$, where $N_{p}$ is the number of selected poles of $G$ within a rectangular bin of area $\delta \alpha \delta E(\delta \alpha=0.02$ and $\delta E=0.05 \varepsilon)$. White circles indicate the positions of band boundaries as expected from the analysis of an infinite system (Figs. 6 and 7). Analogously the white plus symbol marks the expected contact point between the lowest and the first excited band, corresponding to the gap closure.

square lattice inside a circular region of radius $R=26 a$. The finite-size results are compared here with those of an ideal infinite system and the agreement is excellent.
In the evaluation of the DOS in Fig. 8 it is computationally convenient to fix $E$ and, correspondingly, look for values of $\alpha$ satisfying condition (9) or (10). For $E<0$ the $N \times N$ real symmetric matrix $\mathbb{M}^{o}(E)$ and its eigenvalues can be evaluated, thus the values of $\alpha$ solving Eqs. (9) are immediately obtained. For $E>0$ one can use $\tilde{E}=E$ as the starting point to find an approximate solution of the complex Eqs. (10) [15]. We write $z=\tilde{E}+\delta z$ and we assume the first-order expansion $m_{i}^{o}(z)=$ $m_{i}^{o}(\tilde{E})+\delta z m_{i}^{o \prime}(\tilde{E})$ to be valid. By choosing $\alpha=-\operatorname{Re}\left[m_{i}^{o}(\tilde{E})\right]$ each equation reduces to $i \operatorname{Im}\left[m_{i}^{o}(\tilde{E})\right]+\delta z m_{i}^{o^{\prime}}(\tilde{E})=0$, from which $\delta z$ can be directly derived. The energy $E$ and bandwidth $\Gamma$ of the quasi-Bloch states follow from

$$
z=E-i \frac{\hbar}{2} \Gamma \simeq \tilde{E}-i \frac{\operatorname{Im}\left[m_{i}^{o}(\tilde{E})\right]}{m_{i}^{o^{\prime}}(\tilde{E})} .
$$

Note that the derivative $m_{i}^{o^{\prime}}(\tilde{E})$ can be evaluated by resorting to the generalized Hellmann-Feynman theorem: for the complex symmetric matrix $\mathbb{M}^{o}$, one has $d m_{i}^{o}(z) / d z=$ $\vec{u}_{i} \cdot\left[d \mathbb{M}^{o}(z) / d z\right] \vec{u}_{i}$, where $\vec{u}_{i}$ is the right eigenvector of eigenvalue $m_{i}^{o}(z)$, normalized as $\vec{u}_{i} \cdot \vec{u}_{i}=1$ (instead of the usual $\vec{u}_{i} \cdot \vec{u}_{i}^{*}=1$ ).

The first-order approximation (20) is sufficient if $\operatorname{Im}\left[m_{i}^{o}(\tilde{E})\right]$ is small [15], and this is the case for most of the solutions we find. In particular, for quasi-Bloch states one expects a lifetime $\tau=1 / \Gamma \simeq R / v_{g}$, where $R$ is the radius of the region containing the scatterers and $v_{g}$ the group velocity of the state. To verify this behavior we selected a small window on the $[\alpha, E]$ plane, studying the distributions of $\tau$ when the system size varies. The results of this analysis are presented in Fig. 9. We find that the distribution peak follows the expected behavior, confirming that the first-order approximation is sufficient to individuate quasi-Bloch states.

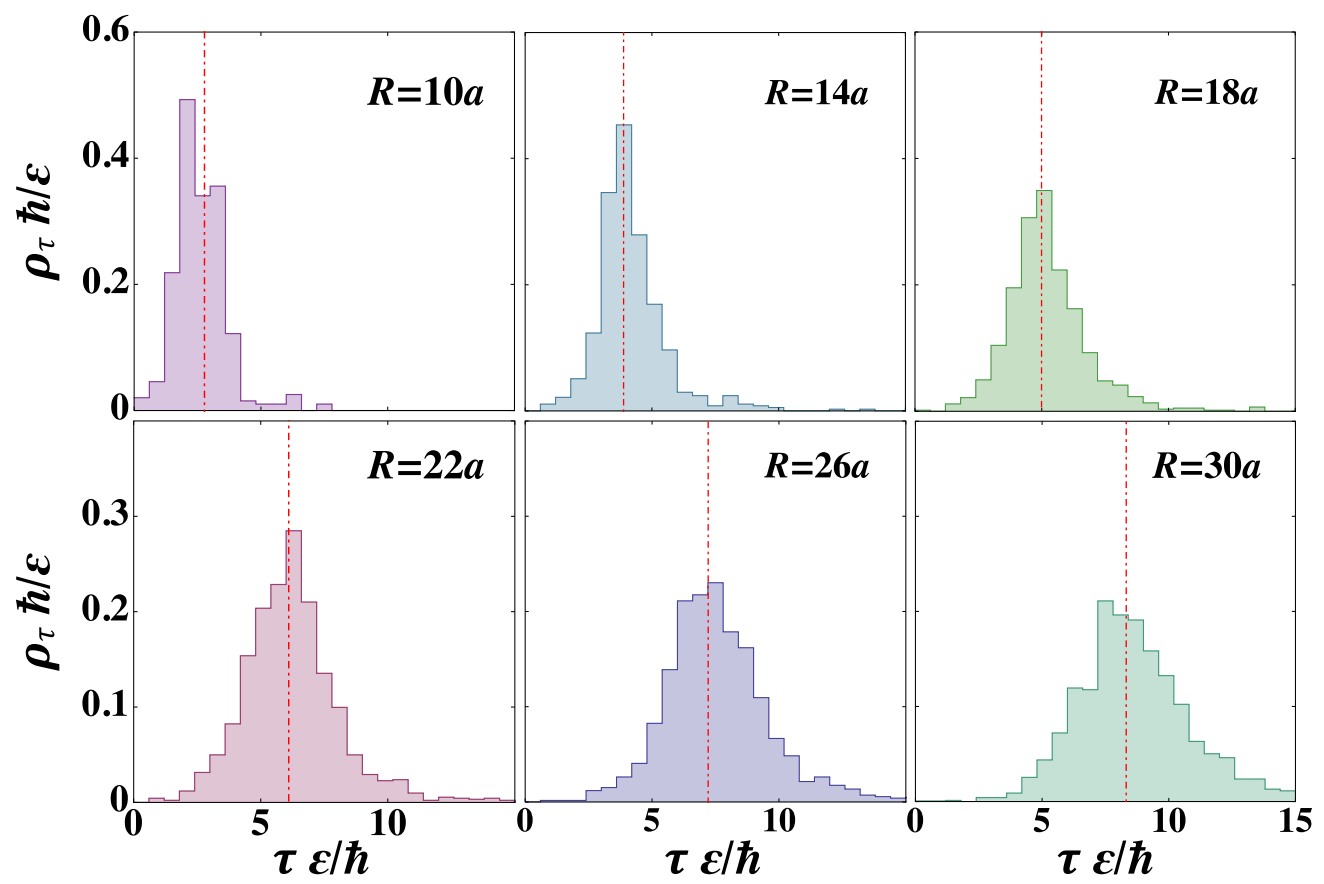

FIG. 9. (Color online) Square lattice of finite size: normalized densities of lifetimes $\tau$ for $E / \varepsilon=6.5 \pm 0.5$ and $\alpha=-0.75 \pm 0.1(\varepsilon=$ $\left.\hbar^{2} / m a^{2}\right)$. Different panels correspond to different radii $R$ of the scattering region. Dot-dashed vertical (red) lines in each panel indicate $R / \bar{v}_{g}$, where $\bar{v}_{g}$ is the average group velocity in the selected region of the $[\alpha, E]$ plane. 


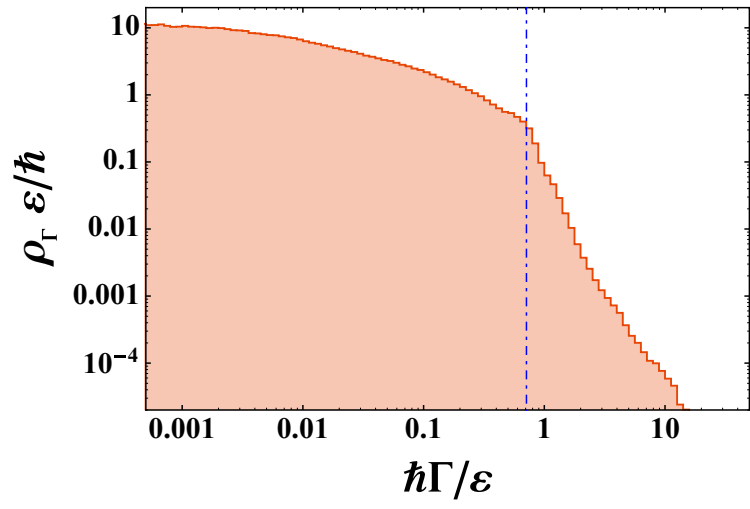

FIG. 10. (Color online) For the same finite-size square lattice considered in Fig. 8: normalized density of bandwidths $\Gamma$ for all positive-energy poles of the Green function $\left(\varepsilon=\hbar^{2} / m a^{2}\right)$. The dotdashed vertical (blue) line at $\Gamma=\Gamma_{\max }$ marks the change in behavior of the density $\rho_{\Gamma}$. Solutions with $\Gamma>\Gamma_{\max }$ have been rejected because they are incompatible with the behavior of a quasi-Bloch bulk state. Here we found $\Gamma_{\max } \simeq 0.7 \varepsilon / \hbar$ (i.e., $\Gamma_{\max } \simeq 2 \mathrm{kHz}$ for a matter wave of ${ }^{87} \mathrm{Rb}$ atoms in an AASL with $a=500 \mathrm{~nm}$ ).

Nevertheless, when the poles of $G$ are evaluated by Eq. (20), one can eventually find some results for which the first order is insufficient. These solutions would present nonphysical negative values of $\Gamma$ and needs to be rejected (in the case presented in Fig. 8 they constitute $10.6 \%$ of positive-energy states). Furthermore, in a finite-size system, states other than quasi-Bloch ones can appear (such as edge states) for which the law $\Gamma \simeq v_{g} / R$ is not valid. In order to tell them apart one can look at the density of $\Gamma$, shown in Fig. 10 for the same system considered in Fig. 8. A neat change in the behavior is found in $\Gamma_{\max } \sim v_{g_{\max }} / R$, where $v_{g_{\max }}$ is the highest estimated group velocity in the range of $E$ and $\alpha$ considered. States with $\Gamma>\Gamma_{\max }$ can in turn be rejected, finally, leaving only solutions behaving as quasi-Bloch bulk states (in the case of Fig. 8 this led to the exclusion of an additional $8.4 \%$ of solutions). A natural question may arise concerning the dependence of $v_{g_{\max }}$ on $\alpha$ and $E$. It has been verified that by considering a cutoff depending on $\alpha$ and $E$ the results are qualitatively the same as those obtained using the aforementioned selection method.

\section{Introduction and effects of disorder}

A remarkable advantage of our atomic artificial lattices with respect to other one-species models is the possibility of naturally introducing disorder in the system. Loading the $B$-atom optical lattice with nonunitary filling would result in the presence of randomly unoccupied sites constituting random defects in the artificial crystal [25]. Our general theoretical approach, presented in Sec. II B, can be used to investigate the effects of this kind of disorder. In particular, we show in Fig. 11 the negative-energy DOS of an AASL of radius $R=32 a$ at different filling factors, obtained fixing $\alpha=-0.75$. In Fig. 11(a), $100 \%$ of the sites are occupied and finite-size effects can be investigated by comparing the DOS with that of the periodic system (presented in Fig. 6). There are no significant discrepancies between the two quantities, confirming the robustness of the results with respect to the

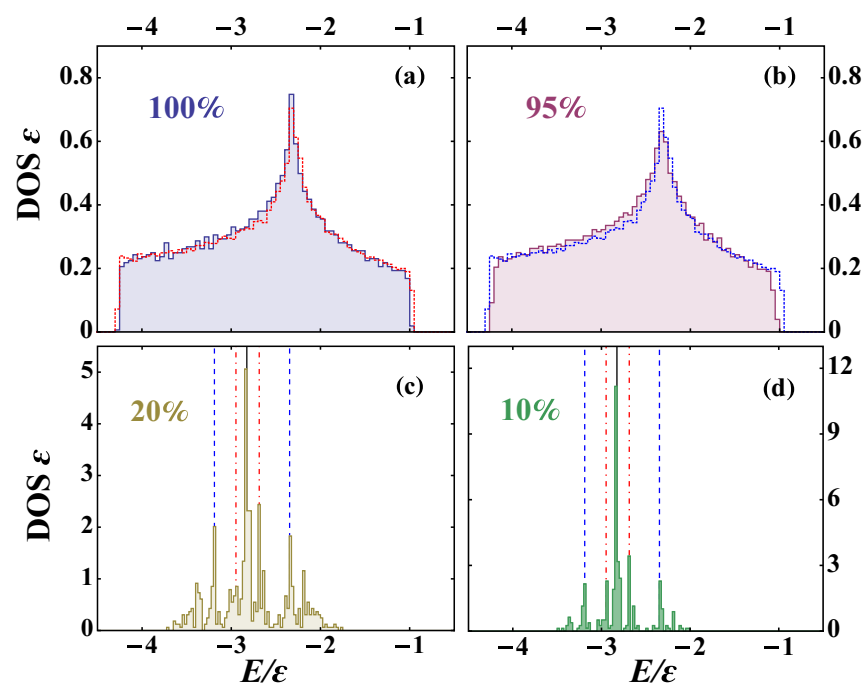

FIG. 11. (Color online) Disordered square lattice: normalized negative-energy DOS at $\alpha=-0.75$ for an AASL of radius $R=32 a$, corresponding to 3209 available lattice sites. Different panels refer to different percentages of randomly occupied sites: (a) 100\%, (b) $95 \%$, (c) $20 \%$, and (d) $10 \%$. Histograms are obtained with a bin size $\delta E=0.05 \varepsilon$ in (a) and (b) and $\delta E=0.025 \varepsilon$ in (c) and (d). Superimposed dotted histograms in (a) and (b) represent the DOS for an infinite periodic system. Vertical lines in (c) and (d) indicate the energies of few-body bound states: $A B$ dimer (solid black line), $A B_{2}$ trimer with $B$ atoms separated by $a$ [dashed (blue) line] and $a \sqrt{2}$ [dot-dashed (red) line]. Here $\varepsilon=\hbar^{2} / m a^{2}$.

system size. The DOS in the presence of $5 \%$ of vacant sites [Fig. 11(b)] appears in turn to be compatible with the results for an ideal periodic system, thus proving robustness also against a small number of vacancies. For a large number of unoccupied sites, instead, the periodicity of the lattice gets lost and the MW interacts locally with few-body clusters of scatterers. As shown in Figs. 11(c) and 11(d), this gives rise to a DOS which is more and more peaked around the energies of few-body $A B_{n}$ bound states. The energies of these bound states can be derived from Eqs. (9), where the eigenvalues of $\mathbb{M}^{o}$ can be analytically obtained for $n \leqslant 4$. The explicit expressions can be found in Eqs. (45) and (46) in Ref. [15], where the emergence of disorder-localized states for low filling is investigated.

\section{B. Triangular lattice}

Another relevant example of an atomic artificial Bravais lattice is the triangular one, whose analysis is also preliminary to the study of intriguing non-Bravais structures, such as graphene and the kagomé lattice. In this case the primitive vectors are $\mathbf{a}_{1}=a(\sqrt{3}, 1) \sqrt{3} / 2$ and $\mathbf{a}_{2}=a(\sqrt{3},-1) \sqrt{3} / 2$, from which the real and reciprocal lattices, illustrated in Fig. 4, are defined.

\section{Infinite system}

To investigate the spectral properties of the atomic artificial triangular lattice (AATL) we need, once again, to solve 

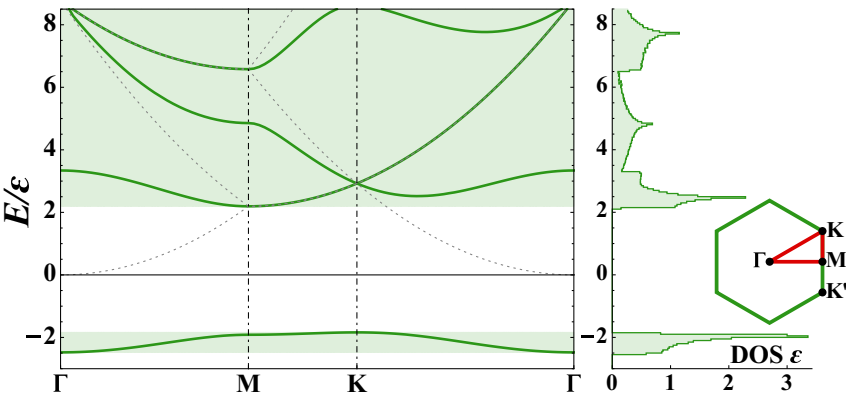

FIG. 12. (Color online) Artificial atomic triangular lattice: band structure (left) and DOS (right) for $\alpha=-0.6$. Left: Behavior of the four lowest energy bands along the $\boldsymbol{\Gamma}-\mathbf{M}-\mathbf{K}-\boldsymbol{\Gamma}$ path (see inset at right). Dashed (gray) lines indicate the dispersion relation for a free matter wave. Right: DOS, as defined in Eq. (19), obtained by evaluating the energy of the bands in $N_{s}=7600$ points sampled inside the aforementioned symmetry path. The bin size of the histogram is $\delta E=0.05 \varepsilon\left(\varepsilon=\hbar^{2} / m a^{2}\right)$.

Eq. (15), now for $C_{\infty} \simeq 0.959662$. We can thus proceed as in Sec. III A 1 for the square lattice.

In Fig. 12 we present a typical spectrum of the system, evaluated in $\alpha=-0.6$. The band structure, studied along the $\boldsymbol{\Gamma}$-M-K- $\boldsymbol{\Gamma}$ high-symmetry path (see inset), shows the presence of a gap, which the DOS confirms to be omnidirectional. Also in this case we verify the existence of a single solution of Eq. (15) between two values of $E_{\text {free }}$. The versatility of this artificial lattice emerges in Fig. 13, where we compare its spectra for different values of the interaction parameter $\alpha$. One finds that there exists a gap of tunable width for $\alpha \lesssim 3.853$, and again, the lowest, isolated band rapidly becomes thin and deep in energy with decreasing $\alpha$.

\section{Finite-size and disorder effects}

For the sake of completeness we again test our results against finite-size effects. With the same method illustrated

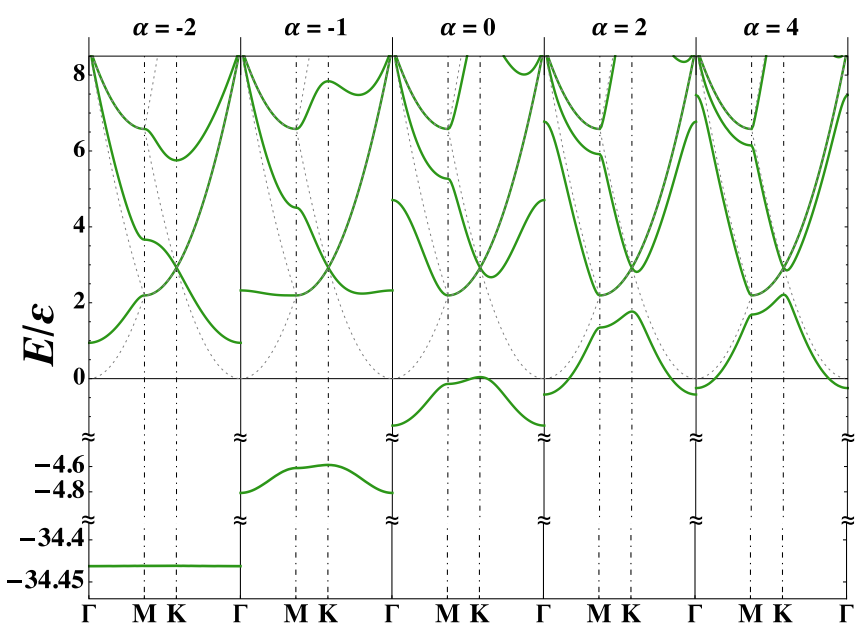

FIG. 13. (Color online) Triangular lattice: comparison of the band structure for different values of the interaction parameter $\alpha$. The spectrum evaluation path is the same as for Fig. 12. Dashed (gray) lines correspond to the energy spectrum of a free matter wave. Energies are normalized on $\varepsilon=\hbar^{2} / m a^{2}$.

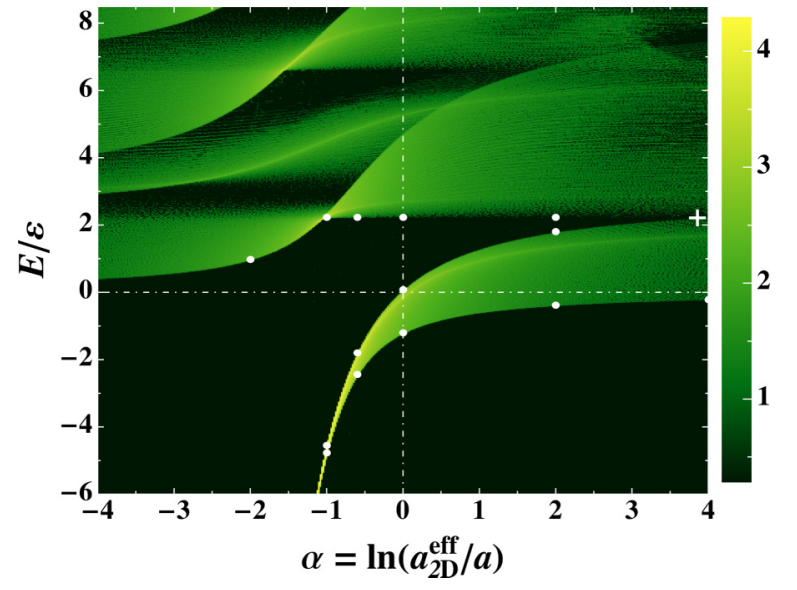

FIG. 14. (Color online) Triangular lattice of finite size: DOS per scatterer in the plane $[\alpha, E / \varepsilon]$ for a system of $N=2125$ scatterers arranged in a triangular lattice inside a disk of radius $R=42 a$. The same method as for Fig. 8 was used, but discretizing $E$ in steps of $0.005 \varepsilon\left(\varepsilon=\hbar^{2} / m a^{2}\right)$. Positive-energy quasi-Bloch bulk states were selected imposing $\Gamma<\Gamma_{\max } \simeq 0.3 \varepsilon / \hbar$ (i.e., $\Gamma_{\max } \simeq 880 \mathrm{~Hz}$ for a matter wave of ${ }^{87} \mathrm{Rb}$ atoms in an AATL with $a=500 \mathrm{~nm}$ ). The color map is applied to the quantity $\log _{10}\left(\frac{N_{p}}{N} \frac{\varepsilon}{\delta \alpha \delta E}\right)$, where $N_{p}$ is the number of selected poles of $G$ within a rectangular bin of area $\delta \alpha \delta E(\delta \alpha=0.02$ and $\delta E=0.025 \varepsilon$ ). White circles indicate the expected positions of the band boundaries as evaluated for the infinite system (Figs. 12 and 13). Analogously, the white plus symbol marks the expected contact point between the lowest and the first excited band.

in Sec. III A 2 we evaluated the DOS on the plane $[\alpha, E]$ for an experimental-size system of $\sim 2100$ scatterers arranged in a triangular lattice inside a circular region of radius $R=42 a$. The results, shown in Fig. 14, are, once again, in good agreement with predictions based on the analysis of an ideal AATL. We also verified that, in analogy to the square-lattice case, the results are robust if a small number of vacancies is randomly introduced in the triangular structure, while few-body states become dominant for low fillings.

\section{NON-BRAVAIS LATTICES}

This section is devoted to generalizing the formalism introduced in Sec. III to the case in which $B$ scatterers are arranged in a non-Bravais lattice: an infinite periodic structure in which a unit cell, now containing $M$ atoms, is repeated to cover the $2 \mathrm{D}$ plane. Such a structure can be equivalently seen as a set of $M$ identical Bravais lattices, the $m$ th and $n$th being displaced by $\mathbf{t}_{m n}$ with respect to each other. The structure will still be invariant if translated by $\mathbf{R} \in L$, for $L=\left\{n_{1} \mathbf{a}_{1}+n_{2} \mathbf{a}_{2}: n_{1}, n_{2} \in \mathbb{Z}\right\}$, where $\mathbf{a}_{1,2}$ are the primitive vectors of a sublattice [cf. Fig. 15(a)]. All the properties of a Bravais lattice remain separately valid for each sublattice. If we denote by $\mathbf{R}_{i}$ the central position of the $i$ th unit cell, and by $\boldsymbol{\rho}_{m}$ the position of the $m$ th atom with respect to this reference, the location of a scatterer in the non-Bravais lattice is given by $\mathbf{r}_{i m}=\mathbf{R}_{i}+\boldsymbol{\rho}_{m}$. The linear system (11) becomes

$$
\sum_{i=1}^{\infty} \sum_{m=1}^{M} \mathbb{M}_{j n, i m} D_{i m}=0
$$



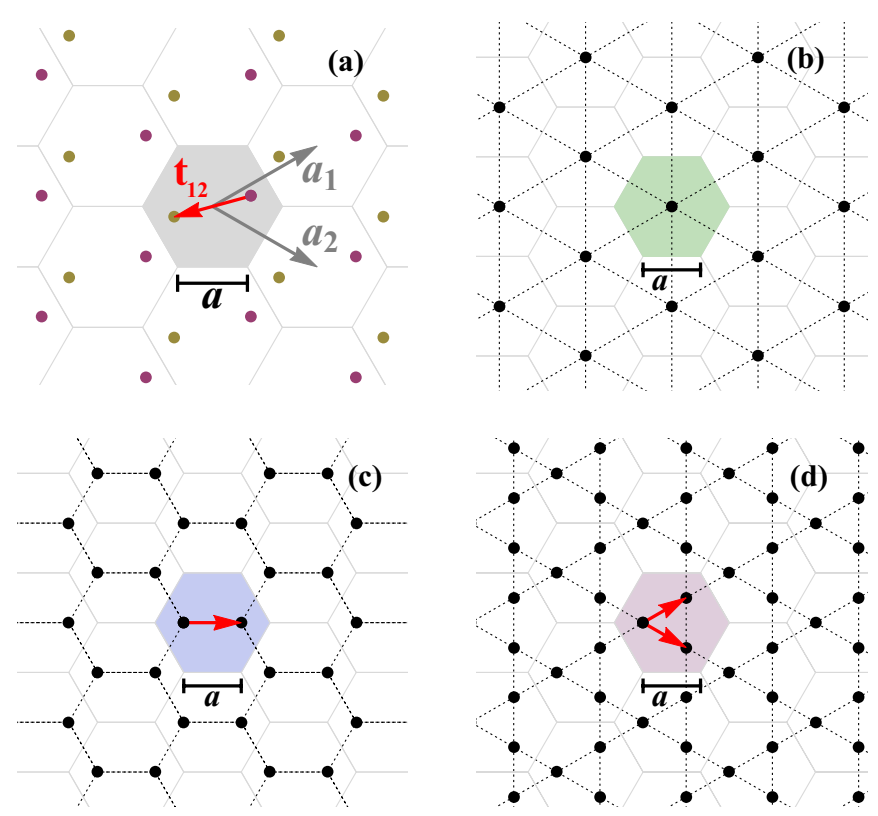

FIG. 15. (Color online) (a) Representation of an $M=2$ nonBravais lattice. It can be seen as a triangular lattice (Fig. 4) with two atoms per unit cell (shaded hexagon) whose relative position is $\mathbf{t}_{12}$. Equivalently, it can be obtained as the superposition of two interpenetrating triangular lattices (distinguished by colors) displaced by $\mathbf{t}_{12}$ with respect to each other. The generating vectors $\mathbf{a}_{1,2}=\frac{3 a}{2}$ (1, $\pm 1 / \sqrt{3}$ ) are also indicated. (b) Representation of the triangular lattice as an example of a basic Bravais lattice, i.e., with one atom per unit cell. The distance between nearest atoms is $a \sqrt{3}$. (c) Representation of the hexagonal lattice of graphene, a two-atom non-Bravais lattice based on the triangular one, obtained for $\mathbf{t}_{12}=\left(\mathbf{a}_{1}+\mathbf{a}_{2}\right) / 3=(a, 0)$. With these definitions, the side of the hexagons has length $a$. (d) Representation of the kagomé lattice, a three-atom non-Bravais lattice based on the triangular one, obtained for $\mathbf{t}_{12}=\mathbf{a}_{1} / 2$ and $\mathbf{t}_{13}=\mathbf{a}_{2} / 2$. The nearest-neighbor distance is $a \sqrt{3} / 2$.

for each $j=1,2, \ldots, \infty$ and $n=1,2, \ldots, M$. Practically, each index has been split with respect to previous notation (i.e., $j \rightarrow j n$ and $i \rightarrow i m$ ) such that the first index runs on the lattice cells, while the second indicates at which of the $M$ sublattices the scatterer belongs. Accordingly, the definition of $\mathbb{M}$ given in Eq. (7) still holds. Bloch's theorem is now separately valid for each sublattice, so that

$$
D_{i m}=D_{j m} e^{i \mathbf{q} \cdot\left(\mathbf{r}_{j m}-\mathbf{r}_{i m}\right)}=D_{j m} e^{i \mathbf{q} \cdot\left(\mathbf{R}_{j}-\mathbf{R}_{i}\right)},
$$

while $D_{i m}$ and $D_{j n}$ stay independent for $m \neq n$ [24]. This assumption makes the present treatment not valid in the few accidental cases in which the non-Bravais lattice degenerates into a Bravais one. For an arbitrary choice of $j$, all the equations in system (21) take the form

$$
\sum_{m=1}^{M} D_{j m} \sum_{i=1}^{\infty} \mathbb{M}_{j n, i m} e^{i \mathbf{q} \cdot\left(\mathbf{R}_{j}-\mathbf{R}_{i}\right)}=0
$$

giving a homogeneous system of $M$ equations in the $M$ unknown $D_{j m}$ 's, whose matrix $\mathbb{T}$ is defined by

$$
\mathbb{T}_{n m}=\sum_{i=1}^{\infty} \mathbb{M}_{j n, i m} e^{i \mathbf{q} \cdot\left(\mathbf{R}_{j}-\mathbf{R}_{i}\right)}
$$

The matrix elements read explicitly

$$
\begin{aligned}
& \mathbb{T}_{n m}=\sum_{\mathbf{R} \in L} \frac{\pi \hbar^{2}}{m} g_{0}\left(\mathbf{R}+\mathbf{t}_{n m}\right) e^{i \mathbf{q} \cdot \mathbf{R}} \text { for } n \neq m, \\
& \mathbb{T}_{n n}=\ln \left(\frac{e^{\gamma}}{2} k a_{2 \mathrm{D}}^{\mathrm{eff}}\right)-i \frac{\pi}{2}+\sum_{\mathbf{R} \in L^{*}} \frac{\pi \hbar^{2}}{m} g_{0}(\mathbf{R}) e^{i \mathbf{q} \cdot \mathbf{R}},
\end{aligned}
$$

with $\mathbf{t}_{n m}=\boldsymbol{\rho}_{n}-\boldsymbol{\rho}_{m}$. The condition $\operatorname{det}(\mathbb{T})=0$ has to be satisfied so that system (23) has a solution, which, in turn, means that this is the condition for the existence of an eigenstate of the $A$-atom MW in the presence of a non-Bravais lattice of $B$ scatterers. Note that the diagonal terms are all the same and that they correspond to the left-hand side of Eq. (13). This naturally implies that for $M=1$ we get back to the case of a Bravais lattice: $\operatorname{det}(\mathbb{T})=\mathbb{T}_{11}=0$ is exactly Eq. (13). It is again convenient to rewrite the sums in terms of reciprocal-lattice vectors. Following the procedure described in the Appendix, we finally have

$$
\begin{aligned}
\mathbb{T}_{n m}= & \frac{2 \pi}{\mathcal{A}} \sum_{\mathbf{K} \in R L} \frac{e^{i(\mathbf{K}-\mathbf{q}) \cdot \mathbf{t}_{n m}}}{k^{2}-|\mathbf{K}-\mathbf{q}|^{2}} \\
\mathbb{T}_{n n}= & C_{\infty}+\ln \left(\frac{e^{\gamma}}{2}\right)+\frac{2 \pi}{\mathcal{A}} \frac{1}{k^{2}-q^{2}} \\
& +\frac{2 \pi}{\mathcal{A}} \sum_{\mathbf{K} \in R L^{*}}\left(\frac{1}{k^{2}-|\mathbf{K}-\mathbf{q}|^{2}}+\frac{1}{K^{2}}\right)+\alpha
\end{aligned}
$$

Besides, from the latter expressions the matrix $\mathbb{T}$ turns out to be explicitly Hermitian.

\section{A. Graphene}

Considering the increasing interest in the intriguing properties of graphene and its artificial realizations [7-9], it seems natural to apply our model to the investigation of the behavior of AAG (cf. [16] for more details): a cold-atom quantum simulator of graphene where, differently from pre-existing models presented in the field [26,27], the periodic potential felt by the MW is generated by other atoms. The honeycomb structure is actually a non-Bravais lattice resulting from the superposition of two interpenetrating triangular ones displaced by $\mathbf{t}=(a, 0)$, as illustrated in Fig. 15(c).

As usual, we begin by considering an ideal infinite system. For a generic two-atom non-Bravais lattice the condition for the existence of an eigenstate is $\operatorname{det}(\mathbb{T})=0$, the matrix $\mathbb{T}$ being a $2 \times 2$ Hermitian one. Once again, the diagonal interaction term $\alpha$ can be isolated by writing $\mathbb{T}=\mathbb{T}^{o}+\mathbb{I} \alpha$, where $\mathbb{I}$ is the $2 \times 2$ identity matrix and

$$
\mathbb{T}^{o}=\mathbb{T}(\alpha=0)=\left(\begin{array}{cc}
f(\mathbf{q}, E) & \varphi(\mathbf{q}, E) \\
\varphi^{*}(\mathbf{q}, E) & f(\mathbf{q}, E)
\end{array}\right),
$$

with $f(\mathbf{q}, E)$ defined as in Eq. (16) and

$$
\varphi(\mathbf{q}, E)=\frac{2 \pi}{\mathcal{A}} \sum_{\mathbf{K} \in R L} \frac{e^{i(\mathbf{K}-\mathbf{q}) \cdot \mathbf{t}}}{k^{2}-|\mathbf{K}-\mathbf{q}|^{2}} .
$$

It follows that condition $\operatorname{det}(\mathbb{T})=0$ is equivalent to

$$
t_{ \pm}^{o}(\mathbf{q}, E)=f(\mathbf{q}, E) \pm|\varphi(\mathbf{q}, E)|=-\alpha
$$




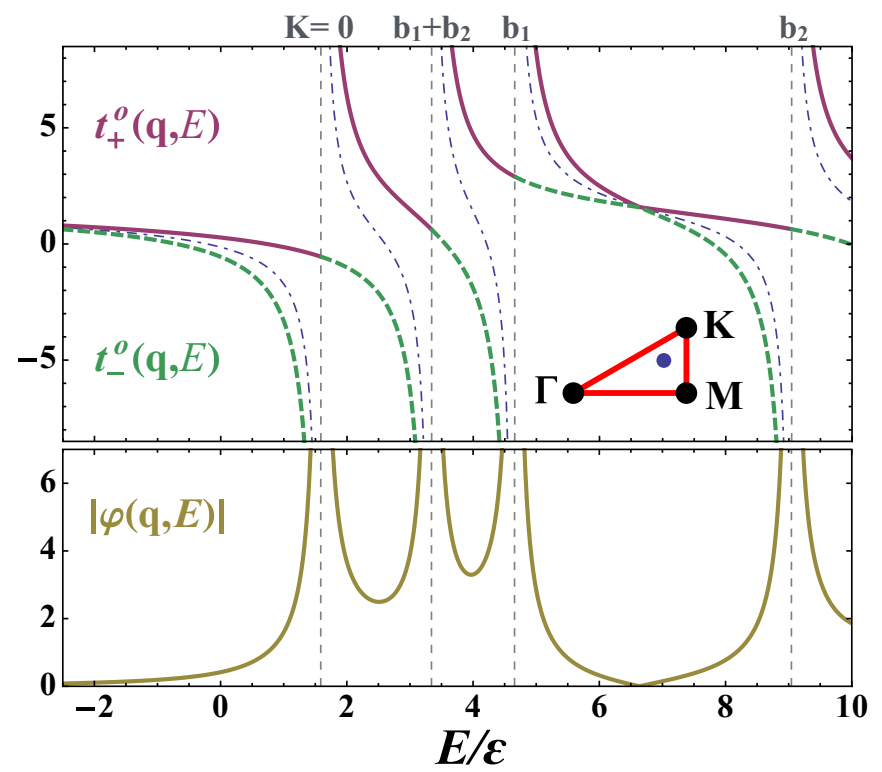

FIG. 16. (Color online) Atomic artificial graphene. Top: Behavior of $t_{+}^{o}(\mathbf{q}, E)$ [solid (purple) line] and $t_{-}^{o}(\mathbf{q}, E)$ [dashed (green) line] as a function of $E$ for $\mathbf{q}=\frac{2 \pi}{3 a}(0.8,0.3)$. Dashed vertical (gray) lines indicate the lowest values of $E_{\text {free }}$ for the selected q. Dot-dashed (blue) curves show the behavior of $f(\mathbf{q}, E)$. Inset: Position of $\mathbf{q}$ within the $\boldsymbol{\Gamma}$-M-K- $\boldsymbol{\Gamma}$ symmetry path (Fig. 4). Bottom: Behavior of $|\varphi(\mathbf{q}, E)|$ for the same q. Here $\varepsilon=\hbar^{2} / m a^{2}$.

where $t_{+}^{o}$ are the two real eigenvalues of $\mathbb{T}^{o}$. It can be easily proved that when $f$ diverges, i.e., when $E \rightarrow E_{\text {free }}$, the absolute value of $\varphi$ exactly cancels the divergence so that the left limit of $t_{+}^{o}$ is finite and equal to the right limit of $t_{-}^{o}$ :

$$
\lim _{E \rightarrow E_{\text {free }}^{\mp}} t_{ \pm}^{o}(\mathbf{q}, E)=\lim _{E \rightarrow E_{\text {free }}} f(\mathbf{q}, E)-\frac{\pi \hbar^{2}}{m \mathcal{A}} \frac{1}{E-E_{\text {free }}} .
$$

A numerical example of this is given in Fig. 16, where the two eigenvalues $t_{ \pm}^{o}(\mathbf{q}, E)$ and $|\varphi(\mathbf{q}, E)|$ are plotted as functions of $E$ for a fixed $\mathbf{q}$. By virtue of the aforementioned properties, only one solution of Eq. (31) exists between two solutions of the corresponding Eq. (15). This practically means that a band of a non-Bravais lattice with $M=2$ is always included between two bands of the corresponding Bravais lattice $(M=1)$.

An example of a spectrum for AAG is presented in Fig. 17 for $\alpha=-0.6$. The band structure and DOS point out the presence of a double gap, which can be manipulated and eventually closed by acting on $\alpha$. A key feature is the existence of two nonequivalent Dirac points, situated in $\mathbf{K}, \mathbf{K}^{\prime}=\frac{2 \pi}{3 a}(1$, $\pm 1 / \sqrt{3}$ ) within the FBZ. Here the two lowest bands touch each other, creating a cone-shaped energy-momentum dispersion. The MW obeys a Dirac-like equation for relativistic massless fermions in which the role of the speed of light is played by $v_{g}$ : the modulus of the group velocity of the wave along the cone. Remarkably, the characteristics of the cones can be manipulated by acting on $\alpha$. At first, for $\alpha \lesssim 0$ the cone lies at negative energies, so that relativistic physics is there played by bound states. Furthermore, similarly to the Bravais artificial lattices, the lowest bands get flat with decreasing $\alpha$, leading to a widening of the cones (cf. Fig. 18). This results in a sensitive decrease in $v_{g}$, even below $1 \mathrm{~mm} / \mathrm{s}$ for

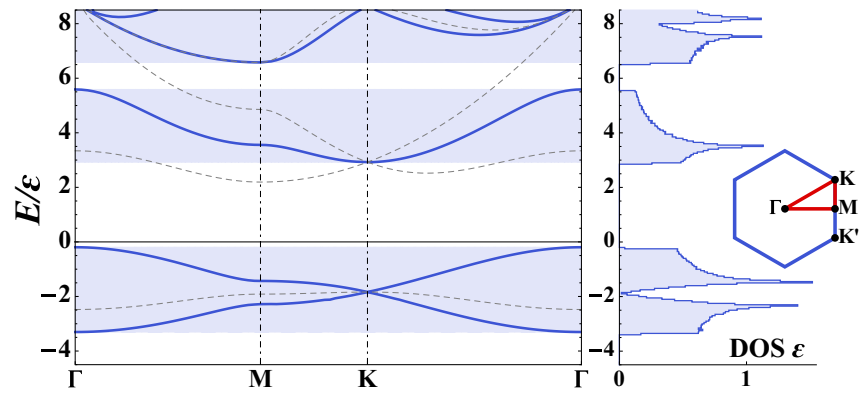

FIG. 17. (Color online) Honeycomb lattice: band structure (left) and DOS (right) for AAG at $\alpha=-0.6$. Left: Behavior of the five lowest energy bands along the $\boldsymbol{\Gamma}-\mathbf{M}-\mathbf{K}-\boldsymbol{\Gamma}$ symmetry path (see inset at right). Dashed (gray) curves show the band structure for the corresponding triangular lattice. Right: DOS [defined in Eq. (19)] obtained by sampling the band energy in $N_{s}=7600$ points within the symmetry path. The histogram bin size is $\delta E=0.05 \varepsilon\left(\varepsilon=\hbar^{2} / m a^{2}\right)$.

typical experimental setups (i.e., up to $10^{-9}$ the corresponding quantity for electrons in real graphene).

Another striking feature emerges at $\alpha=\alpha_{\text {flat }} \simeq-1.631$, a value at which the third isolated band is topologically flat. As shown in Fig. 18, for $\alpha>\alpha_{\text {flat }}$ the third-band maximum is in $\boldsymbol{\Gamma}$ and the minimum in $\mathbf{K}$, while these roles are exchanged for $\alpha<\alpha_{\text {flat }}$. Correspondingly, the band concavity changes at the transition point, leaving the band completely flat around $E \simeq 2.924 \varepsilon\left(\varepsilon=\hbar^{2} / m a^{2}\right)$. It may be argued that, also in the case of Bravais artificial atomic lattices, quasiflat bands emerge for large and negative values of $\alpha$ (see the lowest bands in Figs. 7 and 13), but some important differences exist between these bands and the one we find for AAG. At first, graphene's band is topologically flat, which means that $v_{g}=0$ for every value of q. The quasiflat bands instead keep their structure, even though they are compressed into a small range of energies (this phenomenon is clearly shown in Fig. 7). Moreover, graphene's flat band lies at positive energies, in the propagative region of the MW. The interest in this kind of flat band arises

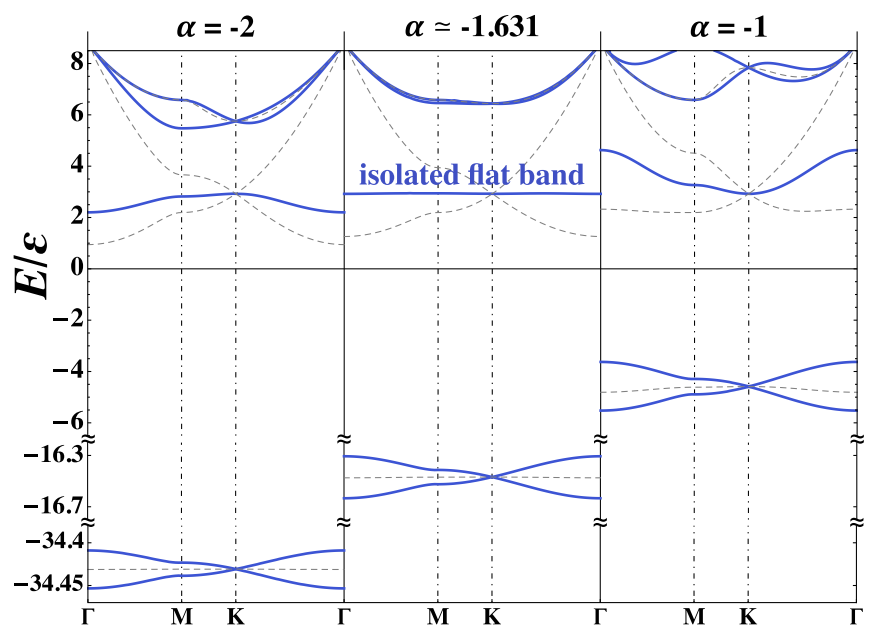

FIG. 18. (Color online) Atomic artificial graphene: comparison of the band structures for different values of $\alpha$ evaluated along the $\boldsymbol{\Gamma}-\mathbf{M}-\mathbf{K}-\boldsymbol{\Gamma}$ symmetry path (see inset in Fig. 17). For $\alpha \simeq-1.631$ the third, isolated band is topologically flat. Dashed (gray) curves show the band structure for the corresponding triangular lattices. The unit of energy is $\varepsilon=\hbar^{2} / m a^{2}$. 
from its nondispersivity: for noninteracting $A$ atoms (the case considered in this work), any MW state would be stationary and localized, the group velocity on the band being strictly 0 . The effects of an eventual $A-A$ interaction, even if extremely small, would be enhanced, leading to the emergence of strongly correlated phases [28]. Nonisolated flat bands have recently been observed in honeycomb lattices for polaritons [29].

Finally, the use of an optical lattice allows us to manipulate and distort the hexagonal structure, thanks to the precise experimental control achievable on the potential landscape. In one-component artificial graphenes such distortion of the structure can lead to the motion and eventual merging of the Dirac cones [30], a phenomenon recently observed experimentally for a Fermi gas in a honeycomb optical lattice [27]. Similarly, such a conductor-to-insulator transition is expected to occur in our AAG. In conclusion, the analysis of finite-size and disorder effects shows the robustness of the aforementioned results against the system size. Dirac cones and flat bands persist for up to $2 \%$ of vacant sites.

\section{B. Kagomé lattice}

As an example of an $M=3$ non-Bravais lattice we consider the kagomé lattice, motivated by the increasing interest in this peculiar structure in which several phenomena of geometric frustration have been predicted [31]. As for graphene, the kagomé lattice is based on the triangular one, but now with three atoms per unit cell forming an equilateral triangle, as depicted in Fig. 15(d).

To study the periodic system, the condition $\operatorname{det}(\mathbb{T})=0$ now translates into looking for solutions of $t_{i}^{o}(\mathbf{q}, E)=-\alpha$, with $i=1,2,3$ and $t_{i}^{o}$ being the three eigenvalues of the matrix $\mathbb{T}^{o}$, defined in Eq. (29). Typical spectra for different values of $\alpha$ are presented in Fig. 19. The two lowest bands again present two Dirac cones for $\mathbf{q}=\mathbf{K}, \mathbf{K}^{\prime}$, but differently from the case of graphene, in the artificial kagomé lattice the third band

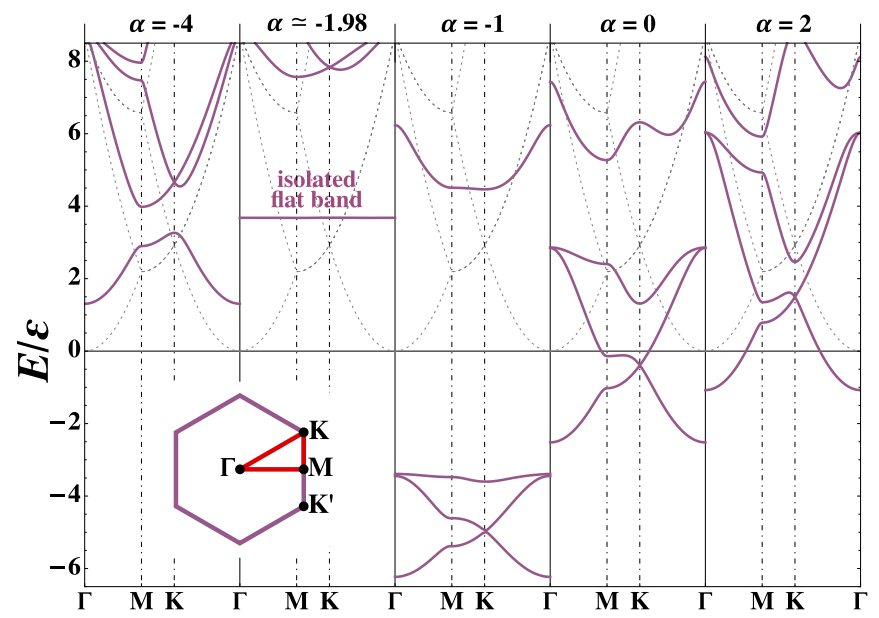

FIG. 19. (Color online) Kagomé lattice: comparison of the band structures of the artificial lattice for different values of $\alpha$ evaluated along the $\boldsymbol{\Gamma}$-M-K- $\boldsymbol{\Gamma}$ symmetry path within the FBZ (see inset). The two lowest bands touch in $\mathbf{q}=\mathbf{K}, \mathbf{K}^{\prime}$, forming a Dirac cone. For $\alpha \simeq$ -1.98 the fourth, isolated band is topologically flat. For $\alpha<-1$ the three lowest bands lie entirely at $E<6.5 \varepsilon\left(\varepsilon=\hbar^{2} / m a^{2}\right)$ and are not shown in the plot. Dashed (gray) lines correspond to the energy spectrum of a free matter wave.

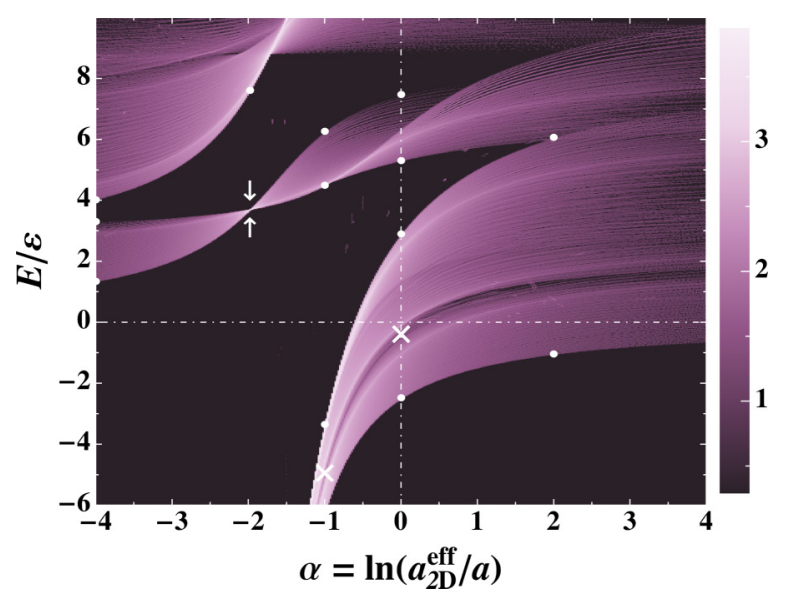

FIG. 20. (Color online) Finite-sized kagomé lattice: DOS per scatterer in the plane $[\alpha, E / \varepsilon]$ for a system of $N=2083$ scatterers arranged in a kagomé lattice inside a disk of radius $R=24 a$. Results were obtained with the same method as described for Fig. 8, discretizing the energies with a step of $0.005 \varepsilon\left(\varepsilon=\hbar^{2} / m a^{2}\right)$. Quasi-Bloch bulk states were selected choosing $\Gamma_{\max } \simeq 0.5 \varepsilon / \hbar$ (i.e., $\Gamma_{\max } \simeq 1460 \mathrm{~Hz}$ for a matter wave of ${ }^{87} \mathrm{Rb}$ atoms for $a=500 \mathrm{~nm}$ ). The color map is applied to the quantity $\log _{10}\left(\frac{N_{p}}{N} \frac{\varepsilon}{\delta \alpha \delta E}\right)$, where $N_{p}$ is the number of selected poles of $G$ within a rectangular bin of area $\delta \alpha \delta E$ $(\delta \alpha=0.02$ and $\delta E=0.025 \varepsilon)$. White $\mathrm{X}$, circles, and arrows indicate, respectively, the positions of Dirac cones, gap boundaries, and the isolated flat band as expected from the analysis of an infinite system (Fig. 19).

moves together with the lowest two. The fourth band is flat and isolated for $\alpha \simeq-2$. We note again that for $|\alpha| \gg 1$ the band structure approaches that of the free MW, as expected the $A-B$ interaction being weak in this limit.

The persistence of the spectral features in systems of experimental size have also been investigated. The density of solutions of Eqs. (9) and (10) for a system with $\sim 2000$ $B$ scatterers is shown in Fig. 20. The agreement with the predictions for an infinite system is extremely good, demonstrating, once again, the robustness of our results against finite-size effects.

\section{CONCLUSIONS}

In this work we have presented a model for the realization of 2D arbitrary AACs based on the use of two independently trapped atomic species. This system revealed itself to be promising as a quantum simulator of bidimensional condensed-matter systems. We have discussed how the interplay between scattering length and trappings allows us to widely tune the interspecies interaction: a key parameter through which the system features can be manipulated. A general theory for finite and infinite periodic systems has been presented, specifying some illustrative examples for both Bravais and non-Bravais lattices. We have proved the emergence of single and multiple gaps, together with the eventual presence of isolated nondispersive flat bands. Furthermore, we have pointed out the existence of Dirac points in AAG and kagomé lattices. The robustness of our results for experimentally realizable systems has been tested against both finite size and disorder. The adaptability of our model makes it suitable for a number of future developments. New perspectives can be 
opened considering also the $p$-wave $A-B$ interaction [19] or the $A$ - $A$ one. The latter, together with the occurrence of flat bands, would make atomic artificial lattices convenient simulators of 2D strongly correlated systems in which nontrivial effects due to geometrical frustration may arise.

\section{APPENDIX: REAL-TO-RECIPROCAL SPACE CHANGE}

This Appendix is devoted to the transformation of sums over real-lattice vectors, appearing in Eqs. (13), (25), and (26), in sums over reciprocal-lattice vectors.

\section{Bravais terms}

We manipulate here the left-hand side of Eq. (13) or, equivalently, a diagonal element of the matrix $\mathbb{T}$ defined in Eq. (26). In particular, we focus on the sum, rewriting $g_{0}(\mathbf{R})$, introduced in Eq. (3), as

$$
g_{0}(\mathbf{R})=\int \frac{d^{2} \mathbf{p}}{(2 \pi)^{2}} \widetilde{g}_{0}(\mathbf{p}) e^{i \mathbf{p} \cdot \mathbf{R}}
$$

in terms of its Fourier transform,

$$
\tilde{g}_{0}(\mathbf{p})=\frac{2 m}{\hbar^{2}}\left(\mathcal{P} \frac{1}{k^{2}-p^{2}}-i \frac{\pi}{2 k} \delta(k-p)\right),
$$

where $\mathcal{P}$ denotes Cauchy's principal value. Once the sum and integral are exchanged we obtain

$$
\sum_{\mathbf{R} \in L^{*}} g_{0}(\mathbf{R}) e^{i \mathbf{q} \cdot \mathbf{R}}=\int \frac{d^{2} \mathbf{p}}{(2 \pi)^{2}} \widetilde{g}_{0}(\mathbf{p})\left(\sum_{\mathbf{R} \in L} e^{i(\mathbf{p}+\mathbf{q}) \cdot \mathbf{R}}-1\right),
$$

where, by adding and subtracting the $\mathbf{R}=0$ term, we now have a sum running over the entire $L$. For such a summation Poisson's identity holds, stating that

$$
\sum_{\mathbf{R} \in L} F(\mathbf{R})=\frac{1}{\mathcal{A}} \sum_{\mathbf{K} \in R L} \widetilde{F}(\mathbf{K}),
$$

where $\mathcal{A}$ is the unit cell area in real space. In the case of interest, $\widetilde{F}(\mathbf{K})=(2 \pi)^{2} \delta^{(2)}(\mathbf{p}+\mathbf{q}-\mathbf{K})$ is the Fourier transform of $F(\mathbf{R})=\exp [i(\mathbf{p}+\mathbf{q}) \cdot \mathbf{R}]$. By integrating out terms involving $\delta$ functions we obtain

$$
\begin{aligned}
\sum_{\mathbf{R} \in L^{*}} \frac{\pi \hbar^{2}}{m} g_{0}(\mathbf{R}) e^{i \mathbf{q} \cdot \mathbf{R}}= & \frac{2 \pi}{\mathcal{A}} \sum_{\mathbf{K} \in R L} \frac{1}{k^{2}-|\mathbf{K}-\mathbf{q}|^{2}} \\
& +i \frac{\pi}{2}-\mathcal{P} \int \frac{d^{2} \mathbf{p}}{2 \pi} \frac{1}{k^{2}-p^{2}} .
\end{aligned}
$$

The principal-valued integral remains to be evaluated. For an arbitrary choice of $\rho$ such that $0<\rho<k$, we have

$$
\mathcal{P} \int \frac{d^{2} \mathbf{p}}{2 \pi} \frac{1}{k^{2}-p^{2}}=\mathcal{P} \int_{p>\rho} \frac{d^{2} \mathbf{p}}{2 \pi} \frac{1}{k^{2}-p^{2}}-\frac{1}{2} \ln \left(1-\frac{\rho^{2}}{k^{2}}\right),
$$

and the Bravais-element $\mathbb{T}_{n n}$ becomes

$$
\begin{aligned}
\mathbb{T}_{n n}= & \ln \left(\frac{e^{\gamma}}{2} k a_{2 \mathrm{D}}^{\mathrm{eff}}\right)+\frac{1}{2} \ln \left(1-\frac{\rho^{2}}{k^{2}}\right)+\frac{2 \pi}{\mathcal{A}} \frac{1}{k^{2}-q^{2}} \\
& +\frac{2 \pi}{\mathcal{A}} \sum_{\mathbf{K} \in R L^{*}} \frac{1}{k^{2}-|\mathbf{K}-\mathbf{q}|^{2}}-\mathcal{P} \int_{p>\rho} \frac{d^{2} \mathbf{p}}{2 \pi} \frac{1}{k^{2}-p^{2}}
\end{aligned}
$$

We now need to introduce two auxiliary quantities,

$\mathcal{S}_{\rho, \mathrm{uv}}=\frac{2 \pi}{\mathcal{A}} \sum_{\mathbf{K} \in R L^{*} \backslash \mathrm{uv}} \frac{1}{k^{2}-|\mathbf{K}-\mathbf{q}|^{2}}-\mathcal{P} \int_{p>\rho \backslash \mathrm{uv}} \frac{d^{2} \mathbf{p}}{2 \pi} \frac{1}{k^{2}-p^{2}}$

and

$$
S_{\rho, \mathrm{uv}}=\frac{2 \pi}{\mathcal{A}} \sum_{\mathbf{K} \in R L^{*} \backslash \mathrm{uv}} \frac{1}{K^{2}}-\int_{p>\rho \backslash \mathrm{uv}} \frac{d^{2} \mathbf{p}}{2 \pi} \frac{1}{p^{2}},
$$

where an arbitrary ultraviolet cutoff (uv) is added in the domains of sum and integration. Note that the second line of Eq. (A7) is exactly $\mathcal{S}_{\rho, \infty}$, which is $\mathcal{S}_{\rho \text {,uv }}$ in the limit of a cutoff boundary pushed to infinity. From definitions (A8) and (A9) it follows that

$$
\begin{aligned}
\mathcal{S}_{\rho, \mathrm{uv}}+S_{\rho, \mathrm{uv}} \underset{\mathrm{uv} \rightarrow \infty}{\longrightarrow} & -\frac{1}{2} \ln \left(\frac{k^{2}}{\rho^{2}}-1\right) \\
& +\frac{2 \pi}{\mathcal{A}} \sum_{\mathbf{K} \in R L^{*}}\left(\frac{1}{k^{2}-|\mathbf{K}-\mathbf{q}|^{2}}+\frac{1}{K^{2}}\right) .
\end{aligned}
$$

We thus add and subtract $S_{\rho, \infty}$ in Eq. (A7), and after some algebraic manipulation we obtain

$$
\begin{aligned}
\mathbb{T}_{n n}= & \ln (\rho a)-S_{\rho, \infty}+\ln \left(\frac{e^{\gamma}}{2}\right)+\frac{2 \pi}{\mathcal{A}} \frac{1}{k^{2}-q^{2}} \\
& +\frac{2 \pi}{\mathcal{A}} \sum_{\mathbf{K} \in R L^{*}}\left(\frac{1}{k^{2}-|\mathbf{K}-\mathbf{q}|^{2}}+\frac{1}{K^{2}}\right)+\alpha,
\end{aligned}
$$

where we have introduced the parameter $\alpha=\ln \left(a_{2 \mathrm{D}}^{\text {eff }} / a\right)$ and the arbitrary unit of length $a$. From Eq. (A11) finally follows the definition of

$$
C_{\infty}=\lim _{\mathrm{uv} \rightarrow \infty} \ln (\rho a)-S_{\rho, \mathrm{uv}},
$$

which numerically converges to a $\rho$-independent quantity determined only by the geometrical properties of the Bravais lattice.

\section{Non-Bravais terms}

The transformation of the off-diagonal element of $\mathbb{T}$ introduced in Eq. (25) follows straightforwardly from the previous one. We again write $g_{0}(\mathbf{R})$ in terms of its Fourier transform $\widetilde{g}_{0}(\mathbf{p})$, obtaining

$$
\begin{aligned}
\sum_{\mathbf{R} \in L} g_{0}\left(\mathbf{R}+\mathbf{t}_{n m}\right) e^{i \mathbf{q} \cdot \mathbf{R}} & =\int \frac{d^{2} \mathbf{p}}{(2 \pi)^{2}} \widetilde{g}_{0}(\mathbf{p}) e^{i \mathbf{p} \cdot \mathbf{t}_{n m}} \sum_{\mathbf{R} \in L} e^{i(\mathbf{p}+\mathbf{q}) \cdot \mathbf{R}} \\
& =\frac{1}{\mathcal{A}} \sum_{\mathbf{K} \in R L} \widetilde{g}_{0}(\mathbf{K}-\mathbf{q}) e^{i(\mathbf{K}-\mathbf{q}) \cdot \mathbf{t}_{n m}},
\end{aligned}
$$

where, in the last step, we have used Poisson's identity as introduced in Eq. (A4). Finally, Eq. (27) directly follows from Eq. (A13) by writing explicitly $\widetilde{g}_{0}(\mathbf{K}-\mathbf{q})$. 
[1] I. Bloch, J. Dalibard, and S. Nascimbène, Nature Phys. 8, 267 (2012).

[2] C. Chin, R. Grimm, P. S. Julienne, and E. Tiesinga, Rev. Mod. Phys. 82, 1225 (2010).

[3] M. Marinescu and L. You, Phys. Rev. Lett. 81, 4596 (1998); Z. Y. Shi, R. Qi, and H. Zhai, Phys. Rev. A 85, 020702(R) (2012); N. Bartolo, D. J. Papoular, L. Barbiero, C. Menotti, and A. Recati, ibid. 88, 023603 (2013).

[4] M. Olshanii, Phys. Rev. Lett. 81, 938 (1998).

[5] M. P. A. Fisher, P. B. Weichman, G. Grinstein, and D. S. Fisher, Phys. Rev. B 40, 546 (1989); M. Greiner, O. Mandel, T. Esslinger, T. W. Hänsch, and I. Bloch, Nature 415, 39 (2002).

[6] K. S. Novoselov, A. K. Geim, S. V. Morozov, D. Jiang, Y. Zhang, S. V. Dubonos, I. V. Gregorieva, and A. A. Firsov, Science 306, 666 (2004).

[7] A. K. Geim and K. S. Novoselov, Nature Mater. 6, 183 (2007).

[8] A. H. Castro Neto, F. Guinea, N. M. R. Peres, K. S. Novoselov, and A. K. Geim, Rev. Mod. Phys. 81, 109 (2009).

[9] M. Polini, F. Guinea, M. Lewenstein, H. C. Manoharan, and V. Pellegrini, Nature Nanotechnol. 8, 625 (2013).

[10] G. Lamporesi, J. Catani, G. Barontini, Y. Nishida, M. Inguscio, and F. Minardi, Phys. Rev. Lett. 104, 153202 (2010).

[11] B. Gadway, D. Pertot, J. Reeves, M. Vogt, and D. Schneble, Phys. Rev. Lett. 107, 145306 (2011)

[12] W. S. Bakr, A. Peng, M. E. Tai, R. Ma, J. Simon, J. I. Gillen, S. Fölling, L. Pollet, and M. Greiner, Science 329, 547 (2010).

[13] F. Nogrette, H. Labuhn, S. Ravets, D. Barredo, L. Béguin, A. Vernier, T. Lahaye, and A. Browaeys, Phys. Rev. X 4, 021034 (2014).

[14] U. Gavish and Y. Castin, Phys. Rev. Lett. 95, 020401 (2005).

[15] M. Antezza, Y. Castin, and D. A. W. Hutchinson, Phys. Rev. A 82, 043602 (2010). [We point out a misprint in Eq. (47), where the factor 8 is missing in front of $\delta^{2}$.]

[16] N. Bartolo and M. Antezza, Europhys. Lett. 107, 30006 (2014).
[17] D. S. Petrov and G. V. Shlyapnikov, Phys. Rev. A 64, 012706 (2001).

[18] P. Massignan and Y. Castin, Phys. Rev. A 74, 013616 (2006).

[19] Y. Nishida and S. Tan, Phys. Rev. Lett. 101, 170401 (2008); Phys. Rev. A 82, 062713 (2010).

[20] N. Bartolo and M. Antezza (unpublished).

[21] M. Olshanii and L. Pricoupenko, Phys. Rev. Lett. 88, 010402 (2001); L. Pricoupenko and M. Olshanii, J. Phys. B 40, 2065 (2007).

[22] C. Kittel, Introduction to Solid State Physics (John Wiley \& Sons, New York, 2004).

[23] I. Carusotto, M. Antezza, F. Bariani, S. De Liberato, and C. Ciuti, Phys. Rev. A 77, 063621 (2008); M. Antezza and Y. Castin, Phys. Rev. Lett. 103, 123903 (2009).

[24] M. Antezza and Y. Castin, Phys. Rev. A 80, 013816 (2009).

[25] M. Antezza and Y. Castin, Phys. Rev. A 88, 033844 (2013).

[26] S.-L. Zhu, B. Wang, and L.-M. Duan, Phys. Rev. Lett. 98, 260402 (2007).

[27] L. Tarruell, D. Greif, T. Uehlinger, G. Jotzu, and T. Esslinger, Nature 483, 302 (2012).

[28] C. Wu, D. Bergman, L. Balents, and S. Das Sarma, Phys. Rev. Lett. 99, 070401 (2007); C. Wu and S. Das Sarma, Phys. Rev. B 77, 235107 (2008).

[29] T. Jacqmin, I. Carusotto, I. Sagnes, M. Abbarchi, D. D. Solnyshkov, G. Malpuech, E. Galopin, A. Lemaître, J. Bloch, and A. Amo, Phys. Rev. Lett. 112, 116402 (2014).

[30] G. Montambaux, F. Piéchon, J.-N. Fuchs, and M. O. Goerbig, Eur. Phys. J. B 72, 509 (2009); L.-K. Lim, J.-N. Fuchs, and G. Montambaux, Phys. Rev. Lett. 108, 175303 (2012); J. IbañezAzpiroz, A. Eiguren, A. Bergara, G. Pettini, and M. Modugno, Phys. Rev. A 88, 033631 (2013).

[31] L. Santos, M. A. Baranov, J. I. Cirac, H.-U. Everts, H. Fehrmann, and M. Lewenstein, Phys. Rev. Lett. 93, 030601 (2004); G.-B. Jo, J. Guzman, C. K. Thomas, P. Hosur, A. Vishwanath, and D. M. Stamper-Kurn, ibid. 108, 045305 (2012). 